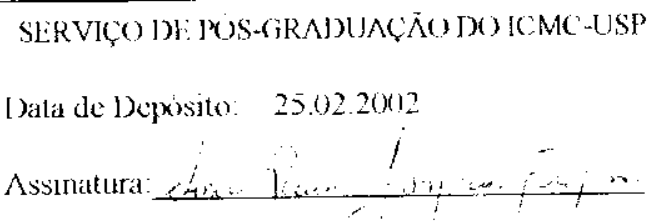

\title{
Bifurcação de Hopf para uma classe de equações diferenciais parciais com retardamento
}

\author{
Katia Andreia Gonçalves de Azevedo
}

Orientador: Prof. Dr. Luiz, Augusto da Costa Ladeira

Tese apresentada ao Instituto de Ciências Matemáticas e de Computação - ICMC-USP. como parte dos requisitos para obtenção do título de Doutor em Ciências - Matematica.

USP - São Carlos

Fevereiro de 2002 


\section{A Comissão Julgadora:}

Prof. Dr. Laiz Ingusto da Costa lakleira

Prof Dr. Plicide Koega Tithos

Prof. Dr. Jasr Silverio dos Samos

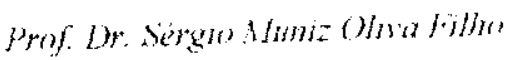

Prof. Dr. (ierson Pesronilho
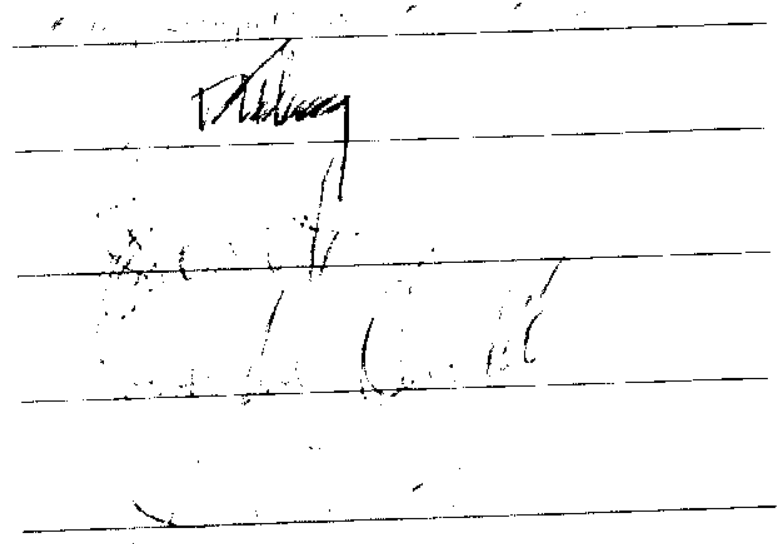
A Deus, a glória. 


\section{Agradecimentos}

Ao Prof. Ladcira pela sua orientação, a qual foi feita com empenho e dedicação, mesmo em momentos difíceis.

Aos professores e colegas do Departamento de Matemática do ICMC-USP pela amizade e apoio, em especial à Sandra, Maria do Carmo e ao Plácido.

Ao Jair por sua atenção e ajuda no desenvolvimento deste trabalho.

Às funcionárias da Secretaria de Pós-Graduação e da Biblioteca pela atenção.

À Marta, Marcia e Selma que compartilharam o meu dia-a-dia.

Ao Mauro pelo seu amor e companherismo.

À minha família: minha mãe Helena, David, Elaine, Marcio, Telma, Neto, Fran e meu querido Lucas.

Aos que oraram por mim.

Enfim, a todos que direta ou indiretamente contribuíram para a realização deste trabalho.

Este trabalho teve suporte financeiro da Fapesp. 
Abstract

In this work we study the retarded reaction-diffusion equation

$$
\left\{\begin{array}{l}
\frac{\partial U}{\partial t}(t, x)=\frac{\partial^{2} U}{\partial x^{2}}(t, x)+k U(t, x)+\frac{k}{\delta} \int_{\cdots \tau}^{-\tau+\delta} g(U(t, x), U(t+s, x) d s \\
U(t, 0)=U(t, \pi)=0, \quad t \geq 0 \\
U(t, x)=\psi(t, x), \quad(t, x) \in[-r, 0] \times[0, \pi] .
\end{array}\right.
$$

We show the existence of a sequence of values $\left\{\tau_{k_{n}}\right\}_{n=0,1,2, \ldots}$ of the parameter $\tau$ such that a Hopf bifurcation occurs when the delay passes through each value $\left\{\tau_{k_{n}}\right\}$.

The main techniques used here are some results on nonlinear eigenvalue problems, the analysis of the characteristic equation of the linearized problem, the Liapunov-Schmidt method and the Implicit Function Theorem. 


\section{Resumo}

Neste trabalho nós estudamos a equação de reação difusão com retardamento

$$
\left\{\begin{array}{l}
\frac{\partial U}{\partial t}(t, x)=\frac{\partial^{2} U}{\partial x^{2}}(t, x)+k U(t, x)+\frac{k}{\delta} \int_{-\tau}^{-\tau+\delta} g(U(t, x), U(t+s, x) d s \\
U(t, 0)=U(t, \pi)=0, \quad t \geq 0 \\
U(t, x)=\psi(t, x), \quad(t, x) \in[-r, 0] \times[0, \pi]
\end{array}\right.
$$

Nós mostramos a existência de uma sequência de valores $\left\{\tau_{k_{n}}\right\}_{n=0,1,2, \ldots}$ do parâmetro $\tau$ tal que uma bifurcação de Hopf ocorre quando o retardo passa através de cada valor $\left\{\tau_{k_{n}}\right\}$.

As técnicas principais usadas aqui são alguns resultados sobre problemas de autovalor não lineares, a análise da equação característica do problema linearizado, o método de Liapunov-Schmidt e o Teorema da Função Implícita. 


\section{Sumário}

Introduçāo

1 Preliminares 4

1 Existência e Unicidade de Equilíbrio Positivo . . . . . . . . . . . . . 4

2 O Método de Liapunov-Schmidt . . . . . . . . . . . . . . . . . . . . . 9

3 Decomposição de $\mathbf{L}^{2}[0, \pi]$ em soma direta . . . . . . . . . . . . . 10

2 O Problema do Autovalor 12

3 Estabilidade do Equílibrio Positivo 24

4 Bifurcação de Hopf $\quad 37$

$\begin{array}{ll}\text { Bibliografia } & 59\end{array}$ 


\section{Introdução}

Equações de reação-difusão com retardo servem como modelos para alguns problemas em ecologia e em dinâmica populacional, como também para sistemas físicos e químicos. Estamos particularmente interessados na seguinte equação de reação-difusão não local com o retardamento incorporado ao controle e condição de fronteira de Dirichlet:

$$
\left\{\begin{array}{l}
\frac{\partial U(t, x)}{\partial t}=\frac{\partial^{2} U(t, x)}{\partial x^{2}}+k U(t, x)+\frac{k}{\delta} \int_{-\tau}^{-\tau+\delta} g(U(t, x), U(t+s, x)) d s \\
U(t, 0)=U(t, \pi)=0, t \geq 0 \\
U(t, x)=\psi(t, x),(t, x) \in[-\tau, 0] \times[0, \pi] \\
\psi \in C\left([-\tau, 0], H_{0}^{1}\right)
\end{array}\right.
$$

com $k, \tau$ e $\delta$ constantes positivas, $0<\delta \leq \tau$.

Esta equação descreve a dinâmica de uma população de espécie única no intervalo $[0, \pi]$, onde $u(t, x)$ representa o tamanho da população em $(t, x)$. Esta condição de fronteira descreve a situação onde o ambientc é cercado por uma região totalmente desfavorável na qual a densidade populacional não pode atingir valores positivos.

Este trabalho é motivado por [1] e [2]. Em [2], Busenberg e Huang estudaram a seguinte equação

$$
\frac{\partial U(t, x)}{\partial t}=\frac{\partial^{2} U(t, x)}{\partial x^{2}}+k U(t, x)[1-U(t-\tau, x)]
$$

com as mesmas condiçōes iniciais e de fronteira.

Eles provaram a existência de soluções periódicas através de bifurcação de Hopf, fizeram uma breve discussão sobre a estabilidade destas soluçōes e interpre- 
taram o modelo biológico que gera esta equação através dos resultados obtidos.

Em [1], Santos e Bená estenderam estes resultados para equações da forma

$$
\frac{\partial U(t, x)}{\partial t}=\frac{\partial^{2} U(t, x)}{\partial x^{2}}+k U(t, x)+k g(U(t, x), U(t-\tau, x))
$$

onde $g: \mathbb{R}^{2} \rightarrow \mathbb{R}$ é dada por $g(a, b)=-a b-a^{3} b^{3}$ e, novamente, as mesmas condiçōes iniciais e de fronteira.

Em nosso caso, estamos considerando o retardo distribuído e observamos que fazendo $\delta \rightarrow 0$, a equaçäo limite é a estuda por Santos e Bená em [1]. A idéia de tomarmos tais equações vem de um trabalho feito por Nussbaum [3], onde controles desta forma são considerados. Também em sua tese de doutoramento, Nicola [15] c [16], considera tal controle.

Para nós, $g: \mathbb{R}^{2} \rightarrow \mathbb{R}$ é uma função de $(a, b)$ e definimos $g(\xi, \xi):=h(\xi)$, $g_{a}(\xi, \xi):=\gamma(\xi)$ e $g_{b}(\xi, \xi):=\eta(\xi)$

As hipóteses que colocamos sobre a função $g$ são as seguintes:

- $g(a, b)$ é uma função continuamente diferenciável, com $g(0,0)=0$,

- $h(\xi)<0$ se $\xi>0$,

- $\frac{h(\xi)}{\xi}$ é decrescente para $\xi>0$,

- $\exists \xi^{*}>0$ tal que $\frac{h\left(\xi^{*}\right)}{\xi^{*}}=-1$,

- $\eta(\xi)<0$ se $\xi>0$,

- $\frac{h(\xi)}{\xi} \geq \gamma(\xi)$ para $\xi>0$,

- $\gamma^{\prime}(0)=-1$ e existe $\eta^{\prime}(0)$, 
$\lim _{(a, b) \rightarrow(0,0)} \frac{g(a, b)}{a b}=-1$

- $\frac{g(a, a)-g(b, b)}{a-b}<\frac{g(a, a)}{a}, a, b>0$

e, como consequência, temos que $\frac{\partial^{2} g(\xi, \xi)}{\partial a \partial b} \rightarrow-1$ quando $\xi \rightarrow 0$.

Observamos que a função $g(a, b)=-a b-\sum_{n=1}^{\infty} \alpha_{n}(a b)^{2 n+1}$, com $\alpha_{n} \geq 0$ satisfaz todas as hipóteses consideradas acima.

Esta tese está desenvolvida da seguinte forma:

No capítulo 1, nós garantiremos a existência e a unicidade de um equilíbrio positivo para a equação (1), bifurcando a partir do equilíbrio nulo, para $k>1$, e acrescentamos alguns pré-requisitos. Observamos que considerando condições de fronteira de Dirichlet, o único equilíbrio espacialmente constante seria a solução nula. Portanto, tais equilíbrios são espacialmente não constantes, dificultando sua análise.

No capítulo 2, nós analisaremos a equação característica associada a equação (1), obtendo a existência de autovalores com parte real nula para $k$ suficientemente próximo de 1.

No capítulo 3 faremos uma descrição do comportamento dos autovalores quando variamos o retardo, obtendo uma descrição completa da estabilidade do equilíbrio positivo, e uma condiçāo para estabilidade assintótica.

Finalmente, no capítulo 4, conseguimos a existência de soluções periódicas através de bifurcação de Hopf tendo como parâmetros $k$ e o retardo $\tau$. 


\section{Capítulo 1}

\section{Preliminares}

\section{Existência e Unicidade de Equilíbrio Positivo}

As soluções equilíbrio de (1) são soluções independentes do tempo mas não espacialmente constantes. Devido à condição de fronteira de Dirichlet, a única solução constante espacialmente ć a solução nula. Dentre estas soluções espacialmente não constantes, estamos interessados nas soluções positivas em $(0, \pi)$.

Uma solução cquilíbrio será uma solução do problema com valor de fronteira não lincar

$$
\left\{\begin{array}{l}
\frac{d^{2} U(x)}{d x^{2}}+k U(x)+\frac{k}{\delta} \int_{-\tau}^{-\tau \mid \delta} g(U(x), U(x)) d s=0 \\
U(0)=U(\pi)=0
\end{array}\right.
$$

que é equivalente a:

$$
\left\{\begin{array}{l}
\frac{d^{2} U(x)}{d x^{2}}+k U(x)+k g(U(x), U(x))=0 \\
U(0)=U(\pi)=0
\end{array}\right.
$$

Os resultados de existência c unicidade para estas soluções de equilíbrio são colocados no seguinte lema, e sua demonstração segue a encontrada em [5], com as devidas adaptações:

Lema 1.1. (i) Para $k \leq 1$, não existe solução positiva em $(0, \pi)$ para (1.2).

(ii) Para $k>1$, existe uma única solução $U_{k}(x)=U(x, k)$ positiva em $(0, \pi)$, de $(1.2)$. 
Prova do item (i): Suponha $k \leq 1$ e que exista uma solução positiva de (1.2), $\tilde{U}(x)$. Entäo $\tilde{U}(x)$ também é soluçäo do problema

$$
\ddot{y}(x)+k y(x)\left[1+\frac{g(\tilde{U}(x), \tilde{U}(x))}{\tilde{U}(x)}\right]=0
$$

$\operatorname{com} y(0)=y(\pi)=0$.

Como, por hipótese, $g(a, a)<0$ se $a>0$, então

$$
k\left[1+\frac{g(\tilde{U}(x), \tilde{U}(x))}{\tilde{U}(x)}\right] \leq 1
$$

Pelo teorema da comparação de Sturm [6, pg. 223], cada solução da equação $\ddot{y}(x)+y(x)=0$ tem pelo menos um zero em $0<x<\pi$. Porém, a função $y=\operatorname{sen} x$ nos fornece uma contradição.

Para provar o item (ii), precisamos de alguns resultados encontrados em [7], que descreveremos a seguir:

Seja $F: \mathcal{E} \rightarrow \mathcal{E}_{1}$, onde $\mathcal{E}$ e $\mathcal{E}_{1}$ são espaços de Banach reais e $F$ é contínua. Suponha que a equação $F(U)=0$ possui uma curva simples de soluções $\mathcal{C}$ dada por $\{U(t) / t \in[a, b]\}$. Se existir $\tau \in(a, b)$ tal que toda vizinhanç̧a de $U(\tau)$ contém pelos menos um zero de $F$ que não está sobre $\mathcal{C}$, dizemos que $U(\tau)$ é um ponto de bifurcação para $F$ com respeito à $\mathcal{C}$.

Uma família especial de tais equações tem a forma

$$
u=G(\lambda, u)
$$

onde $\lambda \in \mathbb{R}, u \in E, E$ é um espaço de Banach real com norma $\|\cdot\|$ e $G: \mathcal{E} \equiv$ $\mathbb{R} \times E \rightarrow E$ é uma transformação compacta e contínua. Em adição,

$$
G(\lambda, u)=\lambda L u+H(\lambda, u)
$$

onde $H(\lambda, u)$ é $O(\|u\|)$ para $u$ próximo da origem, uniformemente para $\lambda$ em intervalos limitados e L é uma transformação linear compacta em $E$. 
Uma solução de (1.3) é um par $(\lambda, u) \in \mathcal{E}$ tal que $u=G(\lambda, u)$. Soluções do tipo $(\lambda, 0)$ são chamadas soluções triviais, $\lambda \in \mathbb{R}$. O fecho do conjunto de soluções não triviais de (1.3) é denotado por $\mathfrak{J}$.

Seja $r(L)$ o conjunto dos $\mu \in \mathbb{R}$ tal que existe $v \in E, v \neq 0$, com $v=\mu L v$, isto é, $r(L)$ consiste dos inversos dos autovalores reais não nulos de $L$.

Sabe-se que os possíveis pontos de bifurcação para (1.3) com respeito a curva de soluções triviais $\{(\lambda, 0) / \lambda \in \mathbb{R}\}$ estão em $\{(\mu, 0) / \mu \in r(L)\}$. De fato, se $\mu \in r(L)$ é de multiplicidade ímpar, $(\mu, 0)$ é um ponto de bifurcação, (veja por ex. [12]).

Seja $\mathcal{O} \subset \mathcal{E}$. Por um contínuo $\mathcal{C}$ de $\mathcal{O}$, entendemos um subconjunto de $\mathcal{O}$ fechado e conexo em $\mathcal{E}$. Dizemos que um contínuo $\mathcal{C}$ em $\mathcal{E}$ encontra o infinito se $\mathcal{C}$ não é limitado.

Temos então o seguinte resultado [7, pag. 490]:

Teorema 1.2. Rabinowitz $S e \mu \in r(L)$ é de multiplicidade impar, então $\mathfrak{J}$ possui um contínuo maximal $\mathcal{C}_{\mu}$ tal que $(\mu, 0) \in \mathcal{C}_{\mu}$ e $\mathcal{C}_{\mu}$ ou

(i) encontra o infinito em $\mathcal{E}$, ou

(ii) encontra $(\hat{\mu}, 0)$, onde $\mu \neq \hat{\mu} \in r(L)$

Por $\mathcal{C}_{\mu}$ maximal entendemos que $\mathfrak{C}_{\mu}$ não é um subcontínuo próprio de qualquer $\mathcal{C} \subset \mathfrak{J}$ com tais propriedades.

Estamos interessados numa aplicação deste resultado a problemas de SturmLiouville não lineares para equações diferenciais ordinárias de segunda ordem. Consideremos

$$
\left\{\begin{array}{l}
\mathcal{L} u \equiv-\left(p u^{\prime}\right)^{\prime}+q u=F\left(x, u, u^{\prime}, \lambda\right), 0<x<\pi \\
C F: a_{0} u(0)+b_{0} u^{\prime}(0)=0, a_{1} u(\pi)+b_{1} u^{\prime}(\pi)=0
\end{array}\right.
$$

onde CF são as condições de fronteira e $\left(a_{0}^{2}+b_{0}^{2}\right)\left(a_{1}^{2}+b_{1}^{2}\right) \neq 0$.

A função $F(x, \xi, \eta, \lambda)=\lambda a(x) \xi+H(x, \xi, \eta, \lambda)$ e $H$ é $\left.O\left(\left(\xi^{2}+\eta^{2}\right)\right)^{\frac{1}{2}}\right)$, próximo a $(\xi, \eta)=(0,0)$ uniformemente para $\lambda$ em intervalos limitados.

As funções $p, q$, c a são, respectivamente, $C^{1}$ e positiva, contínua, contínua e positiva em $[0, \pi]$, e $F$ é contínua em scus argumentos em $[0, \pi] \times \mathbb{R}^{3}$. 
Seja $E$ o espaço de Banach

$$
C^{1}[0, \pi] \cap C . F .=\left\{u \in C^{1}[0, \pi] / a_{0} u(0)+b_{0} u^{\prime}(0)=0, a_{1} u(\pi)+b_{1} u^{\prime}(\pi)=0\right\}
$$

com a norma usual

$$
\|u\|_{1}=\max _{x \in[0, \pi]}|u(x)|+\max _{x \in[0, \pi]}\left|u^{\prime}(x)\right|
$$

e considercmos os seguintes conjuntos:

$S_{n}^{+}=\{u \in E / \mathrm{u}$ tem exatamente $n-1$ zeros nodais em $(0, \pi)$ e $u(x)>0$ para $x$ próximo a 0$\}$. Por um zero nodal entendemos que a funçāo troca de sinal neste zero e por um zero nodal simples, quando a derivada da função é não nula neste zero ;

$$
\begin{aligned}
& S_{n}^{-}=-S_{n}^{+} \text {e } S_{n}=S_{n}^{+} \cup S_{n}^{-} \\
& \mathcal{E}=\mathbb{R} \times E, \mathfrak{J}_{n}^{+}=\mathbb{R} \times S_{n}^{+}, \mathfrak{J}_{n}^{--}=\mathbb{R} \times S_{n}^{-} \text {e } \mathfrak{J}_{n}=\mathbb{R} \times S_{n}
\end{aligned}
$$

O seguintc resultado acontece para a equação (1.4), como uma aplicação do teorema (1.2), também encontrado em [7, pag. 501].

Teorema 1.3. Para cada inteiro $n>0$ e cada $\nu=+$ ou -, existe urn contínuo de soluções da equação (1.4) em $\mathfrak{J}_{n}^{\nu} \cup\left\{\left(\lambda_{n}, 0\right)\right\}$ que encontra $\left(\lambda_{n}, 0\right)$ e o $\infty$ em $\mathcal{E}$.

Observação 1.4. Aqui $\lambda_{n}, n>0$ são os autovalores de $\mathcal{L} u$.

Prova do item (ii) Para aplicar a teoria descrita, observamos primeiramente, que a equação (1.2) pode ser vista como o problema considerado acima:

$$
\left\{\begin{array}{l}
-\ddot{U}(x)=k U(x)+k g(U(x), U(x)) \\
U(0)=U(\pi)=0
\end{array}\right.
$$

com $p \equiv 1, q \equiv 0, a \equiv 1$ e $F(x, \xi, k)=k \xi+k g(\xi, \xi)$, continua em todos os seus argumentos. Então $H(\xi, k)=k g(\xi, \xi)$ e $H$ é obviamente $O(\|\xi\|)$ quando $\|\xi\| \rightarrow 0$, uniformemente para $k$ em intervalos limitados.

Os autovalores de $-\ddot{U}$ são inteiros positivos, da forma $\lambda=\alpha^{2}, \alpha \in \mathbb{Z}$.

Então, pelo teorema (1.3), existe um contínuo de soluções da equação (1.2) em $\mathfrak{J}_{1}^{+} \cup\left\{\left(k_{1}, 0\right)\right\}$ que encontra $\left(k_{1}, 0\right)$ e $\infty$ em $\mathcal{E}$, com $k_{1}=1$. 
Agora, seja $U(x)>0$ para $0<x<\pi$ uma tal solução. Se $x_{0}$ é ponto de máximo, então $\ddot{U}\left(x_{0}\right)<0$ e assim, $\frac{g\left(U\left(x_{0}\right), U\left(x_{0}\right)\right)}{U\left(x_{0}\right)}$ deve satisfazer

$$
-1<\frac{g\left(U\left(x_{0}\right), U\left(x_{0}\right)\right)}{U\left(x_{0}\right)}<0
$$

Como por hipótese, $\frac{g(a, a)}{a}$ é decrescente se $a>0$ c existe $a^{*} \in \mathbb{R}, a^{*}>0$ tal que $\frac{g\left(a^{*}, a^{*}\right)}{a^{*}}=-1$, então, $U\left(x_{0}\right)<a^{*}$. Portanto, $U(x)<a^{*}$ para todo $x \in[0, \pi]$, sendo desta forma limitada por $a^{*}$. Como este raciocínio vale para toda $U \in \mathfrak{J}_{1}^{+}$, esta limitação é uniforme e sendo este contínuo ilimitado, se prolonga para todo $k>1$.

Para concluirmos a prova do item (ii), resta provarmos a unicidade da solução positiva.

Suponha que $U_{1}$ e $U_{2}$ são duas soluções positivas distintas da equação (1.2), então $U_{1}-U_{2}$ é solução do problema

$$
\ddot{y}+k\left[1+\frac{g\left(U_{1}, U_{1}\right)-g\left(U_{2}, U_{2}\right)}{U_{1}-U_{2}}\right] y=0
$$

com condição de fronteira $y(0)=y(\pi)=0$.

Por hipótese

$$
\frac{g\left(U_{1}, U_{1}\right)-g\left(U_{2}, U_{2}\right)}{U_{1}-U_{2}}<\frac{g\left(U_{1}, U_{1}\right)}{U_{1}}
$$

Logo, toda solução do problema

$$
\ddot{y}+k\left[1+\frac{g\left(U_{1}, U_{1}\right)}{U_{1}}\right] y=0
$$

tem um zero entre 0 e $\pi$, inclusive $U_{1}$, o que é uma contradiçäo. 


\section{O Método de Liapunov-Schmidt}

Uma ferramenta básica para o estudo de bifurcação de Hopf é o método de Liapunov-Schmidt, ou método alternativo, que expomos a scguir. Exposições detalhadas podem ser encontradas em Golubitsky e Schaeffer [17] e Knops [18].

Sejam $X, Y$ espaços de Banach, $L: \operatorname{Dom}(L) \subset X \rightarrow Y$ um operador linear e $N: X \rightarrow Y$ um operador (não necessariamente linear). Estamos interessados em estudar a equação

$$
L x=N x, x \in \operatorname{Dom}(L) .
$$

Vamos supor satisfeita a seguinte hipótese:

$\left(\mathrm{H}_{0}\right)$ existem projeções $P: X \rightarrow X$ e $Q: Y \rightarrow Y$ tais que $R(P)=N(L)$ e $R(I-Q)=R(L)$.

É imediato que a equação (1.6) é equivalente ao sistema

$$
\left\{\begin{array}{l}
(I-Q)(L x-N x)=0 \\
Q(L x-N x)=0
\end{array}\right.
$$

A hipótese $\left(\mathrm{H}_{0}\right)$ implica que $(I-Q) L x=L x \in Q L x=0$ para todo $x \in \operatorname{Dom}(L)$ e, portanto, o sistema (1.7) é equivalente a

$$
\left\{\begin{array}{l}
L x=(I-Q) N x \\
Q N x=0
\end{array}\right.
$$

Da hipótese $\left(\mathrm{H}_{0}\right)$ temos ainda que $\tilde{L}=L \mid \operatorname{Dom}(L) \cap R(I-P)$ ć um isomorfismo algébrico entre $\operatorname{Dom}(L) \cap R(I-P)$ e $R(L)$, e tem um inverso $K=\tilde{L}^{-1}$ definido em $R(L)$, ou seja, $L K=I$ em $R(L)$ e $K L=I-P$ em $\operatorname{Dom}(L)$.

Substituindo estas relações em (1.8), temos provado o seguinte teorema:

Teorema 1.5. Suponhamos satisfeita a hipótese $\left(\mathrm{H}_{0}\right)$. Então existe um operador linear $K: R(L) \rightarrow X$ tal que $L K=I$ em $R(L), K L=I-P$ em $\operatorname{Dom}(L)$ e a equação (1.6) é equivalente ao sistema 


$$
\left\{\begin{array}{l}
\text { (a) } x=P x+K(I-Q) N x \\
\text { (b) } Q N x=0
\end{array}\right.
$$

A equação $(1.9$ (a)) e(1.9 (b)) são chamadas equação auxiliar e equação de bifurcação, respectivamente.

Se $\operatorname{Dom}(L)$ for um subespaço fechado de $X$ e se $L$ for um operador contínuo, então $\operatorname{Dom}(\tilde{L})=\operatorname{Dom}(L) \cap R(I-P)$ ć fechado e $\tilde{L}$ é contínuo. Assim, do teorema do gráfico fechado, seguc-se que $K$ é contínuo. Se, além disso, $N$ for de classe $C^{1}$ em alguma vizinhança da origem com $N(0)=0$ e $D N(0)=0$, então a função $f: \operatorname{Dom}(\tilde{L}) \times N(L) \rightarrow Y$

$$
f(x, w)=x-w+K(I-Q) N x
$$

tem as seguintes propriedades: $f(0,0)=0$ c $D_{1} f(0,0)=I$, a identidade de $\operatorname{Dom}(\tilde{L})$. Pelo Teorcma da Função Implícita segue que a equação $f(x, w)=0$ pode ser resolvida numa vizinhança de $(x, w)=(0,0)$ fornecendo uma única solução $x=x^{*}(w)$. É imediato que $x^{*}(w)$ satisfaz $x^{*}(w)=w+K(I-Q) N x^{*}(w)$.

Então, o problema de encontrar soluçōes $x$ para a cquação (1.6) tica reduzido ao de encontrar soluçōes $w$ para a equação (1.9 (b)) com $x$ substituido por $x^{*}(w)$, isto é, o problema é o de encontrar as soluções da equação

$$
Q N x^{*}(w)=0
$$

A existência de uma solução $w$ em $R(P)$ para a equação (1.10) é equivalente à existência de uma solução de (1.6). Em vista disso, costuma-se dizer que a equação (1.10) constitui um problema alternativo para a cquação (1.6).

\section{Decomposição de $\mathbf{L}^{2}[0, \pi]$ em soma direta}

Consideremos a equação $\ddot{y}+y=f$, com $f \in L^{2}[0, \pi]$. A solução geral desta equação é dada por:

$$
y(t)=\left(a-\int_{0}^{t} f(s) \operatorname{sen}(s) d s\right) \cos t+\left(b+\int_{0}^{t} f(s) \cos s d s\right) \operatorname{sen} t
$$


Se considerarmos as condições iniciais $y(0)=y(\pi)=0$, temos, fazendo $t=0$ que $a=0 \mathrm{e}$, fazendo $t=\pi$, que $\int_{0}^{\pi} f(s) \operatorname{sen} s d s=0$. Assim,

Teorema 1.6. O problema de contorno

$$
\left\{\begin{array}{l}
\ddot{y}+y=f, \quad f \in L^{2}[0, \pi] \\
y(0)=y(\pi)=0
\end{array}\right.
$$

tem solução se, e somente se, $\int_{0}^{\pi} f(s)$ sen $s d s=0$.

Dito de outro modo, $f \in R\left(D^{2}+1\right)$ com a condição de fronteira $y(0)=y(\pi)=0$ se, e somente se, $\int_{0}^{\pi} f(s) \operatorname{sen} s d s=0$, isto é, $\langle f, \operatorname{sen}(\cdot)\rangle=0$.

É claro que, $\ddot{y}+y=0$ tem como solução $y(t)=a \cos t+b$ sen $t$, e, portanto, considerando as mesmas condições de fronteira, $y \in N\left(D^{2}+1\right)$ se, e somente se, $y(t)=b \operatorname{sen} t$, ou seja, $N\left(D^{2}+1\right)=\operatorname{span}[\operatorname{sen}(\cdot)]$.

Pelo Teorema da Projeção, temos que

$$
L^{2}[0, \pi]=N\left(D^{2}+1\right) \oplus R\left(D^{2}+1\right)
$$

considerando as condiçôes de fronteira $y(0)=y(\pi)=0$. 


\section{Capítulo 2}

\section{O Problema do Autovalor}

Sejam $k>1$ e $U_{k}$ o equilíbrio positivo de (1). Para estudarmos tanto a estabilidade do equilíbrio positivo quanto a bifurcação de Hopf, precisamos de uma análise da equação característica.

Para simplificar a notação nós indicaremos $g\left(U_{k}, U_{k}\right)=g\left(U_{k}\right)$. Então, a linearizaçāo de (1) em torno de $U_{k}$ é:

$$
\left\{\begin{array}{l}
\frac{\partial}{\partial t} V(t, x)=\frac{\partial^{2}}{\partial x^{2}} V(t, x)+k\left(1+g_{a}\left(U_{k}\right)\right) V(t, x)+\frac{k}{\delta} \int_{-\tau}^{-\tau+\delta} g_{b}\left(U_{k}\right) V(t+s, x) d s, t>0 \\
V(t, 0)=V(t, \pi)=0, t \geq 0 \\
V(t, x)=\phi(t, x),(t, x) \in[-\tau, 0] \times[0, \pi]
\end{array}\right.
$$

onde $\phi \in C([-\tau, 0], X)=C, \operatorname{com} X=L^{2}[0, \pi]$.

Se nós introduzirmos o operador $A(k): \operatorname{Dom}(A(k)) \subset X \rightarrow X$ dado por:

$$
A(k)=D^{2}+k\left(1+g_{a}\left(U_{k}\right)\right)
$$

onde $D^{2}=\frac{\partial^{2}}{\partial x^{2}}, \operatorname{Dom}(A(k))=H^{2} \cap H_{0}^{1}$, então $A(k)$ é o gerador infinitesimal de um $C_{0}$-semigrupo compacto (veja [8]) c podemos escrever (2.1) como uma equação diferencial funcional semilinear abstrata:

$$
\left\{\begin{array}{l}
\dot{V}(t)=A(k) V(t)+\frac{k}{\delta} \int_{-\tau}^{-\tau+\delta} g_{b}\left(U_{k}\right) V(t+s) d s, t>0 \\
V(t)=\phi(t), t \in[-\tau, 0] ; \phi \in C
\end{array}\right.
$$


$\operatorname{com} V(t)=V(t, \cdot)$ e $\phi(t)=\phi(t, \cdot)$

À equação (2.2) associamos um semigrupo chamado semigrupo solução, definido por $U(t): C \rightarrow C$,

$$
U(t) \phi=u_{t}(\phi), \quad t \geq 0
$$

onde $u_{t}(\phi)$ denota a solução de (2.2) sujeita a condiçāo inicial $u_{0}=\phi$.

$\{U(t)\}_{t \geq 0}$ é um $C_{0}$-semigrupo em $C$ e definimos por $A_{\tau}(k): \operatorname{Dom}\left(A_{\tau}(k)\right) \subset$ $C \rightarrow C$, o gerador infinitesimal do semigrupo induzido pelas soluções da equação (2.2) com

$$
A_{\tau}(\phi)=\dot{\phi}
$$

$\operatorname{Dom}\left(A_{\tau}(k)\right)=\left\{\phi \in C \cap C^{1}, \phi(0) \in H_{0}^{1} \cap H^{2}, \dot{\phi}(0)=A(k) \phi(0)+\frac{k}{\delta} \int_{-\tau}^{-\tau+\delta} g_{b}\left(U_{k}\right) \phi(s) d s\right\}$

É através da caracterização do espectro pontual de $A_{\tau}(k), \sigma\left(A_{\tau}(k)\right)$, que obtemos informações sobre a estabilidade de $U_{k}$. Os pontos de $\mathbb{C}$ que pertencem ao espectro pontual de $A_{\tau}(k)$ são raízes da equação característica associada à (2.2):

$$
\Delta(k, \lambda, \tau) y=0, y \in H_{0}^{1} \cap H^{2}, y \neq 0
$$

onde

$$
\Delta(k, \lambda, \tau)=D^{2}+k\left(1+g_{a}\left(U_{k}\right)\right)+\frac{k}{\delta} \int_{-\tau}^{-\tau+\delta} g_{b}\left(U_{k}\right) e^{\lambda s} d s-\lambda
$$

ou equivalentemente,

$$
\Delta(k, \lambda, \tau)=D^{2}+k\left(1+g_{a}\left(U_{k}\right)\right)+K g_{b}\left(U_{k}\right) e^{-\lambda \tau}\left[\frac{e^{\lambda \delta}-1}{\lambda \delta}\right]-\lambda
$$

Como os autovalores de $A_{\tau}(k)$ são funçōes contínuas de $\tau$ (veja [4]), aqueles valores de $\tau$ para os quais $\sigma\left(A_{\tau}(k)\right)$ contém um autovalor imaginário puro são importantes na análise da estabilidade e bifurcação de soluçōes periódicas. 
O operador $A_{\tau}(k)$ tem um autovalor imaginário puro $\lambda=i \omega, \omega \neq 0$, para algum $\tau>0$ se, e somente se, a equação

$$
\left[A(k)+k g_{b}\left(U_{k}\right) e^{i \omega \tau}\left(\frac{e^{i \omega \delta}-1}{i \omega \delta}\right)-i \omega\right] y=0,0 \neq y \in H^{2} \cap H_{0}^{1},
$$

tem solução para algum valor de $\omega>0, \tau \in[0,2 \pi)$. Se nós encontrarmos um par $(\omega, \tau)$ tal que a equação (2.4) tern uma solução não nula em $H^{2} \cap H_{0}^{1}$, então, temos que $\Delta\left(k, i \omega, \tau_{n}\right)=0, \tau_{n}=(\theta+2 n \pi) / \omega, n=0,1,2, \ldots$, e assim, a sequência $\tau_{n}$ é uma possível candidata para que ocorra bifucarção de Hopf.

Através de algums lemas, provaremos que se $0<k-1<<1$, existe um único par $(\omega, \theta)$ que resolve:

$$
\left[A(k)+k g_{b}\left(U_{k}\right) e^{i \omega \theta}\left(\frac{e^{i \omega \delta}-1}{i \omega \delta}\right)-i \omega\right] y=0,0 \neq y \in H^{2} \cap H_{0}^{1}
$$

Primeiramente, vamos decompor o espaço $X$ em soma direta do núcleo de $D^{2}+1$ pela imagem desta aplicação, [ver teorema (1.6)], isto é,

$$
L^{2}[0, \pi]=N\left(D^{2}+1\right) \oplus R\left(D^{2}+1\right)
$$

onde $N\left(D^{2}+1\right)=\operatorname{span}[\operatorname{sen}(\cdot)] \mathrm{c}$

$$
R\left(D^{2}+1\right)=\left\{y \in L^{2}[0, \pi] ;\langle\operatorname{sen}(\cdot), y\rangle=\int_{0}^{\pi} \operatorname{sen}(x) y(x) d x=0\right\}
$$

Agora, projetando o equilíbrio positivo nestes subespaços, podemos encontrar e estimar $U_{k}$.

Lema 2.1. (i) Existe $k^{*}>1$ e uma transformação continuamente diferenciável $k \rightarrow\left(\xi_{k}, \alpha_{k}\right)$ de $\left[1, k^{*}\right]$ em $\left(H^{2} \cap H_{0}^{1}\right) \cap R\left(D^{2}+1\right) \times \mathbb{R}^{+}$tal que

$$
\begin{gathered}
U_{k}=\alpha_{k}(k-1)\left[\operatorname{sen}(\cdot)+(k-1) \xi_{k}\right], k \in\left[1, k^{*}\right], \\
\alpha_{1}=\frac{\int_{0}^{\pi} \operatorname{sen}^{2}(x) d x}{\int_{0}^{\pi} \operatorname{sen}^{3}(x) d x} e
\end{gathered}
$$




$$
\xi_{1}=-\left(D^{2}+1\right)^{-1}\left[\operatorname{sen}(\cdot)\left(1-\alpha_{1} \operatorname{sen}(\cdot)\right)\right], \quad\left\langle\operatorname{sen}(\cdot), \xi_{1}\right\rangle=0
$$

$\operatorname{com} \xi_{1} \in H^{2} \cap H_{0}^{1}$.

(ii) Se $(\omega, \theta, y)$ resolve (2.5) com $0 \neq y \in H^{2} \cap H_{0}^{1}$, entäo $\frac{\omega}{k-1}$ é uniformemente limitada para $k \in\left[1, k^{*}\right]$, e

$$
\omega\langle y, y\rangle=-k \frac{(\cos (\theta-\omega s)-\cos (\theta))}{\omega s}\left\langle g_{b}\left(U_{k}\right) y, y\right\rangle
$$

(iii) Se $z \in H^{2} \cap H_{0}^{1} e\langle\operatorname{sen}(\cdot), z\rangle=0$ então $\left|\left\langle\left(D^{2}+1\right) z, z\right\rangle\right| \geq 3\|||\|_{L^{2}}^{2}$.

Prova: (i) Seja $h:\left(H^{2} \cap H_{0}^{1}\right) \times \mathbb{R} \times \mathbb{R} \rightarrow L^{2} \times \mathbb{R}$ definida por:

$h(\xi, \alpha, k)=\left(\left(D^{2}+1\right) \xi+(1+\alpha k \Upsilon(\xi, \alpha, k)(\operatorname{sen}(\cdot)+(k-1) \xi))(\operatorname{sen}(\cdot)+(k-1) \xi),\langle\operatorname{sen}(\cdot), \xi\rangle\right)$ onde

$$
\Upsilon(\xi, \alpha, k)=\frac{g\left(\alpha_{k}(k-1)(\operatorname{sen}(\cdot)+(k-1) \xi), \alpha_{k}(k-1)(\operatorname{sen}(\cdot)+(k-1) \xi)\right)}{\alpha_{k}^{2}(k-1)^{2}(\operatorname{sen}(\cdot)+(k-1) \xi)^{2}}
$$

Observemos que $\Upsilon\left(\xi_{1}, \alpha_{1}, 1\right)=-1$, usando a hipótese que

$$
\lim _{(a, b) \rightarrow(0,0)} \frac{g(a, b)}{a b}=-1 \text {. }
$$

Com a definição de $\xi_{1}$, verificamos que

$$
h\left(\xi_{1}, \alpha_{1}, 1\right)=\left(\left(D^{2}+1\right) \xi_{1}+\left(1-\alpha_{1} \operatorname{sen}(\cdot)\right) \operatorname{sen}(\cdot),\left\langle\operatorname{sen}(\cdot), \xi_{1}\right\rangle\right)=(0,0)
$$

\section{Calculamos}

$$
D_{(\xi, \alpha)} h\left(\xi_{1}, \alpha_{1}, 1\right)(\xi, \alpha)=\left(\left(D^{2}+1\right) \xi-\alpha \operatorname{sen}^{2}(\cdot),\langle\operatorname{sen}(\cdot), \xi\rangle\right)
$$


e vemos que $D_{(\xi, \alpha)} h\left(\xi_{1}, \alpha_{1}, 1\right)$ é bijetora de $\left(H^{2} \cap H_{0}^{1}\right) \times \mathbb{R} \rightarrow L^{2} \times \mathbb{R}$. De fato, $D_{(\xi, \alpha)} h\left(\xi_{1}, \alpha_{1}, 1\right)(\xi, \alpha)=(0,0)$ se, e somente se, $\langle\operatorname{sen}(\cdot), \xi\rangle=0$ e $\left(D^{2}+1\right) \xi=$ $\alpha \operatorname{sen}^{2}(\cdot)$. Como $\operatorname{sen}^{2}(\cdot) \notin R\left(D^{2}+1\right)$, então $\alpha=0$. Daí, $\xi \in N\left(D^{2}+1\right) \cap R\left(D^{2}+1\right)$, o que nos diz que $\xi=0$. Portanto $D_{(\xi, \alpha)} h\left(\xi_{1}, \alpha_{1}, 1\right)$ é injetora. A sobrejetividade segue do fato que dado $\eta \in R\left(D^{2}+1\right)$ e $\beta \in \mathbb{R}$, existe uma solução $(\xi, \alpha)$ do problema

$$
\begin{gathered}
\left(D^{2}+1\right) \xi-\alpha \operatorname{sen}^{2}(\cdot)=\eta \\
\langle\operatorname{sen}(\cdot), \xi\rangle=\beta
\end{gathered}
$$

Segue do teorema da função implícita que existe $k^{*}>1$ e uma transformação continuamente diferenciável $k \rightarrow\left(\xi_{k}, \alpha_{k}\right) \in\left(H^{2} \cap H_{0}^{1}\right) \cap R\left(D^{2}+1\right) \times \mathbb{R}^{+}$para $k \in\left[1, k^{*}\right]$ tal que

$$
h\left(\xi_{k}, \alpha_{k}, k\right)=0, \quad k \in\left[1, k^{*}\right]
$$

e usando este fato, provamos que $W_{k}=\alpha_{k}(k-1)\left[\operatorname{sen}(\cdot)+(k-1) \xi_{k}\right]$ é solução de (1.2). Pela unicidade de solução temos que $U_{k}=W_{k}$, para $k \in\left[1, k^{*}\right]$

(ii) Pela equação característica temos:

$$
\left\langle\left[A(k)+k g_{b}\left(U_{k}\right) e^{-i \omega \theta}\left(\frac{e^{i \omega \delta}-1}{i \omega \delta}\right)-i \omega\right] y, y\right\rangle=0
$$

Pelo fato de $A(k)$ ser auto-adjunta, a igualdade acima torna-se:

$$
\omega\langle y, y\rangle=-k\left(\frac{\cos (\theta-\omega \delta)-\cos (\theta)}{\omega \delta}\right)\left\langle g_{b}\left(U_{k}\right) y, y\right\rangle
$$

Em norma:

$$
\frac{|\omega|}{(k-1)}|\langle y, y\rangle|=\left|-k\left(\frac{\cos (\theta-\omega \delta)-\cos (\theta)}{\omega \delta}\right)\right|\left|\left\langle\frac{g_{b}\left(U_{k}\right)}{(k-1)} y, y\right\rangle\right|
$$




$$
\begin{aligned}
\frac{|\omega|}{(k-1)} & =\frac{|k|\left|\left(\frac{\cos (\theta-\omega \delta)-\cos (\theta)}{\omega \delta}\right)\right|\left|\left\langle\frac{g_{b}\left(U_{k}\right)}{(k-1)} y, y\right\rangle\right|}{\|y\|_{L^{2}}^{2}} \\
& \leq k^{*} M\left|\left(\frac{\cos (\theta-\omega \delta)-\cos (\theta)}{\omega \delta}\right)\right| \frac{\|y\|_{L^{2}}^{2}}{\|y\|_{L^{2}}^{2}} \\
& \leq k^{*} M
\end{aligned}
$$

onde $M$ vem do fato que $\lim _{k \rightarrow 1} \frac{g_{b}\left(U_{k}\right)}{(k-1)}$ existe, garantido pela hipótese sobre $\eta^{\prime}(0)$.

(iii) Demonstração feita em [2].

Agora, queremos escrever a cquação (2.5), como um sistema de equações.

Para cada $k \in\left[1, k^{*}\right]$, suponha que $(\omega, \theta, y)$ é uma solução de $(2.5)$ com $0 \neq$ $y \in H^{2} \cap H_{0}^{1}$.

Ignorando um fator escalar, $y$ pode ser representado por

$$
y=\beta \operatorname{sen}(\cdot)+(k-1) z\langle\operatorname{sen}(\cdot), z\rangle=0, \beta \geq 0
$$

E queremos que para $k$ próximo de 1 a igualdade abaixo esteja satisfeita

$$
\|y\|_{L^{2}}^{2}=\beta^{2}\|\operatorname{sen}(\cdot)\|_{L^{2}}^{2}+(k-1)^{2}\|z\|_{L^{2}}^{2}=\|\operatorname{sen}(\cdot)\|_{L^{2}}^{2}
$$

Chamando $\omega=(k-1) \rho$ e substituindo junto com a expressão para $y$ em $(2.5)$, temos:

$$
\begin{aligned}
& {\left[A(k)+k g_{b}\left(U_{k}\right) e^{-i \theta}\left(\frac{e^{i \omega \delta}-1}{i \omega \delta}\right)-i \omega\right] y=} \\
& =\left[D^{2}+k\left(1+g_{a}\left(U_{k}\right)\right)+k g_{b}\left(U_{k}\right) e^{-i \theta}\left(\frac{e^{i \omega \delta}-1}{i \omega \delta}\right)-i \omega\right](\beta \operatorname{sen}(\cdot)+(k-1) z)= \\
& =-\beta \operatorname{sen}(\cdot)+(k-1) D^{2} z+k \beta \operatorname{sen}(\cdot)+k(k-1) z+(\beta \operatorname{sen}(\cdot)+(k-1) z)\left[k \left(g_{a}\left(U_{k}\right)+\right.\right. \\
& \left.\left.g_{b}\left(U_{k}\right) e^{-i \theta}\left(\frac{e^{i \omega \delta}-1}{i \omega \delta}\right)\right)-i \omega\right]=
\end{aligned}
$$




$$
\begin{aligned}
& \quad=(k-1)\left\{\frac{-\beta \operatorname{sen}(\cdot)}{(k-1)}+D^{2} z+z-z+\frac{k \beta \operatorname{sen}(\cdot)}{(k-1)}+k z+(\beta \operatorname{sen}(\cdot)+(k-1) z)\left[\frac { k } { ( k - 1 ) } \left(g_{a}\left(U_{k}\right)+\right.\right.\right. \\
& \left.\left.\left.g_{b}\left(U_{k}\right)\left(\frac{e^{i \omega \delta}-1}{i \omega \delta}\right) e^{-i \theta}\right)-i \rho\right]\right\}= \\
& \quad=(k-1)\left\{\left(D^{2}+1\right) z+(\beta \operatorname{sen}(\cdot)+(k-1) z)\left[\frac{k}{(k-1)}\left[g_{a}\left(U_{k}\right)+g_{b}\left(U_{k}\right) e^{-i \theta}\left(\frac{e^{i \omega \delta}-1}{i \omega \delta}\right)\right]-\right.\right. \\
& i \rho+1]\}= \\
& \quad=(k-1) h_{1}(z, \beta, \rho, \theta, k)
\end{aligned}
$$

Portanto, (2.5) é equivalente ao sistema:

$$
\left\{\begin{array}{l}
h_{1}(z, \beta, \rho, \theta, k)=0 \\
h_{2}(z)=\operatorname{Re}(\langle\operatorname{sen}(\cdot), z\rangle)=0 \\
h_{3}(z)=\operatorname{Im}(\langle\operatorname{sen}(\cdot), z\rangle)=0 \\
h_{4}(z, \beta, k)=\left(\beta^{2}-1\right)\|\operatorname{sen}(\cdot)\|_{L^{2}}^{2}+(k-1)^{2}\|z\|_{L^{2}}^{2}=0
\end{array}\right.
$$

Vamos calcular $\lim _{k \rightarrow 1} \frac{\omega}{(k-1)}$, isto é, $\rho_{1}$ :

$$
\begin{aligned}
& \frac{\omega}{(k-1)}\langle y, y\rangle=-\frac{k}{(k-1)}\left(\frac{\cos (\theta-\omega \delta)-\cos (\theta)}{\omega \delta}\right)\left\langle g_{b}\left(U_{k}\right) y, y\right\rangle \\
& \frac{\omega}{(k-1)}\langle y, y\rangle=-k\left(\frac{\cos (\theta-\omega \delta)-\cos (\theta)}{\omega \delta}\right)\left\langle\frac{g_{b}\left(U_{k}\right)}{U_{k}} \frac{U_{k}}{k-1} y, y\right\rangle
\end{aligned}
$$

Fazendo $y=\beta \operatorname{sen}(\cdot)+(k-1) z$

$$
\frac{\omega}{(k-1)}\langle\beta \operatorname{sen}(\cdot)+(k-1) z, \beta \operatorname{sen}(\cdot)+(k-1) z\rangle=
$$$$
=-k\left(\frac{\cos (\theta-\omega \delta)-\cos (\theta)}{\omega \delta}\right)\left\langle\frac{g_{b}\left(U_{k}\right)}{U_{k}} \alpha_{k}\left(\operatorname{sen}(\cdot)+(k-1) \xi_{k}\right)(\beta \operatorname{sen}(\cdot)+(k-1) z), \beta \operatorname{sen}(\cdot)+\right.
$$
$(k-1) z\rangle$

Quando $k \rightarrow 1$ 


$$
\begin{aligned}
& \lim _{k \rightarrow 1} \frac{\omega}{(k-1)}\langle\beta \operatorname{sen}(\cdot), \beta \operatorname{sen}(\cdot)\rangle=-\operatorname{sen}(\theta) \lim _{k \rightarrow 1} \frac{g_{b}\left(u_{k}\right)}{U_{k}}\left\langle\alpha_{1} \beta \operatorname{sen}^{2}(\cdot), \beta \operatorname{sen}(\cdot)\right\rangle= \\
& =-\operatorname{sen}(\theta) \lim _{k \rightarrow 1} \frac{g_{b}\left(u_{k}\right)}{U_{k}}\langle\beta \operatorname{sen}(\cdot), \beta \operatorname{sen}(\cdot)\rangle \\
& \quad \text { Portanto, } \lim _{k \rightarrow 1} \frac{\omega}{(k-1)}=-\operatorname{sen}(\theta) \lim _{k \rightarrow 1} \frac{g_{b}\left(u_{k}\right)}{U_{k}} .
\end{aligned}
$$

Também podemos verificar que

$$
\begin{aligned}
\lim _{k \rightarrow 1} \frac{1}{(k-1)}\left[g_{a}\left(U_{k}\right)+g_{b}\left(U_{k}\right) e^{-i \theta}\left(\frac{e^{i \omega \delta}-1}{i \omega \delta}\right)\right] & =-\alpha_{1} \operatorname{sen}(\cdot)+\lim _{k \rightarrow 1} \frac{g_{b}\left(u_{k}\right)}{U_{k}} \alpha_{1} \operatorname{sen}(\cdot) e^{-i \theta} \\
& =-\alpha_{1}\left(1-\lim _{k \rightarrow 1} \frac{g_{b}\left(u_{k}\right)}{U_{k}} e^{-i \theta}\right) \operatorname{sen}(\cdot)
\end{aligned}
$$

observando que $\lim _{k \rightarrow 1} \frac{e^{i \omega \delta}-1}{i \omega \delta}=1$.

Teorema 2.2. Se $0<k^{*}-1<<1$, então existe uma transformação continuamente diferenciável de $\left[1, k^{*}\right]$ em $H^{2} \cap H_{0}^{1} \times \mathbb{R}^{3}, k \rightarrow\left(z_{k}, \beta_{k}, \rho_{k}, \theta_{k}\right)$ tal que

$$
\begin{gathered}
z_{1}=\left(1+i \lim _{k \rightarrow 1} \frac{g_{b}\left(u_{k}\right)}{U_{k}}\right) \xi_{1} \\
\beta_{1}=1, \quad \theta_{1}=\frac{\pi}{2} \\
\rho_{1}=-\lim _{k \rightarrow 1} \frac{g_{b}\left(u_{k}\right)}{U_{k}}
\end{gathered}
$$

$e\left(z_{k}, \beta_{k}, \rho_{k}, \theta_{k}\right)$ resolve (2.6) para $k \in\left[1, k^{*}\right]$, com $\xi_{1}$ dado no lema (2.1).

Além disso, se $k \in\left[1, k^{*}\right]$ e $\left(z^{k}, \beta^{k}, \rho^{k}, \theta^{k}\right)$ resolve $(2.6)$ para $k \in\left[1, k^{*}\right]$, com $\rho^{k}>0$ $e \theta \in[0,2 \pi)$, então $\left(z^{k}, \beta^{k}, \rho^{k}, \theta^{k}\right)=\left(z_{k}, \beta_{k}, \rho_{k}, \theta_{k}\right)$.

Prova:Definamos $H:\left(H^{2} \cap H_{0}^{1}\right) \times \mathbb{R}^{3} \times \mathbb{R} \rightarrow L^{2} \times \mathbb{R}^{3}$ por:

$$
H=\left(h_{1}, h_{2}, h_{3}, h_{4}\right)
$$

Mostremos que $H\left(z_{1}, \beta_{1}, \rho_{1}, \theta_{1}, 1\right)=0$ 


$$
\begin{aligned}
& \quad h_{1}\left(z_{1}, \beta_{1}, \rho_{1}, \theta_{1}, 1\right)=\left(D^{2}+1\right) z_{1}+\beta_{1} \operatorname{sen}(\cdot)\left[-\alpha_{1}\left(1-\lim _{k \rightarrow 1} \frac{g_{b}\left(u_{k}\right)}{U_{k}} e^{-i \theta_{1}}\right) \operatorname{sen}(\cdot)-\right. \\
& \left.i\left(-\operatorname{sen}\left(\theta_{1}\right) \lim _{k \rightarrow 1} \frac{g_{b}\left(u_{k}\right)}{U_{k}}\right)+1\right]= \\
& \quad=-\left(1+i \lim _{k \rightarrow 1} \frac{g_{b}\left(u_{k}\right)}{U_{k}}\right) \operatorname{sen}(\cdot)\left[1-\alpha_{1} \operatorname{sen}(\cdot)\right]-\alpha_{1}\left(1+i \lim _{k \rightarrow 1} \frac{g_{b}\left(u_{k}\right)}{U_{k}}\right) \operatorname{sen}^{2}(\cdot)+\operatorname{sen}(\cdot)+ \\
& i \lim _{k \rightarrow 1} \frac{g_{b}\left(u_{k}\right)}{U_{k}} \operatorname{sen}(\cdot)= \\
& \quad=-\left(1+i \lim _{k \rightarrow 1} \frac{g_{b}\left(u_{k}\right)}{U_{k}}\right) \operatorname{sen}(\cdot)\left[1-\alpha_{1} \operatorname{sen}(\cdot)\right]+\left(1+i \lim _{k \rightarrow 1} \frac{g_{b}\left(u_{k}\right)}{U_{k}}\right) \operatorname{sen}(\cdot)\left[1-\alpha_{1} \operatorname{sen}(\cdot)\right]= \\
& 0
\end{aligned}
$$

Observemos que:

$$
\left\langle\operatorname{sen}(\cdot), z_{1}\right\rangle=\left\langle\operatorname{sen}(\cdot),\left(1+i \lim _{k \rightarrow 1} \frac{g_{b}\left(u_{k}\right)}{U_{k}}\right) \xi_{1}\right\rangle=\left\langle\operatorname{sen}(\cdot), \xi_{1}\right\rangle+i \lim _{k \rightarrow 1} \frac{g_{b}\left(u_{k}\right)}{U_{k}}\left\langle\operatorname{sen}(\cdot), \xi_{1}\right\rangle
$$

Assim,

$$
\begin{aligned}
& h_{2}\left(z_{1}\right)=\operatorname{Re}\left(\left\langle\operatorname{sen}(\cdot), z_{1}\right\rangle\right)=\left\langle\operatorname{sen}(\cdot), \xi_{1}\right\rangle=0 \\
& h_{3}\left(z_{1}\right)=\operatorname{Im}\left(\left\langle\operatorname{sen}(\cdot), z_{1}\right\rangle\right)=\lim _{k \rightarrow 1} \frac{g_{b}\left(u_{k}\right)}{U_{k}}\left\langle\operatorname{sen}(\cdot), \xi_{1}\right\rangle=0
\end{aligned}
$$

É óbvio que se $k=1$ e $\beta=1, h_{4}\left(z_{1}, \beta_{1}, 1\right)=0$.

Portanto, $H\left(z_{1}, \beta_{1}, \rho_{1}, \theta_{1}, 1\right)=0$

Agora, seja $T=\left(T_{1}, T_{2}, T_{3}, T_{4}\right):\left(H^{2} \cap H_{0}^{1}\right) \times \mathbb{R}^{3} \rightarrow L^{2} \times \mathbb{R}^{3}$, definida por $T=D_{(z, \beta, \rho, \theta)} H\left(z_{1}, \beta_{1}, \rho_{1}, \theta_{1}, 1\right)$. Então,

$$
\begin{aligned}
& T_{1}(z, \beta, \rho, \theta)=\left(D^{2}+1\right) z+\beta \operatorname{sen}(\cdot)\left(1+i \lim _{k \rightarrow 1} \frac{g_{b}\left(u_{k}\right)}{U_{k}}\right)\left(1-\alpha_{1} \operatorname{sen}(\cdot)\right)-i \operatorname{sen}(\cdot) \rho- \\
& \theta \alpha_{1} \lim _{k \rightarrow 1} \frac{g_{b}\left(u_{k}\right)}{U_{k}} \operatorname{sen}^{2}(\cdot) \\
& T_{2}(z)=\operatorname{Re}\langle\operatorname{sen}(\cdot), z\rangle
\end{aligned}
$$




$$
\begin{aligned}
& T_{3}(z)=\operatorname{Im}\langle\operatorname{sen}(\cdot), z\rangle \\
& T_{4}(\beta)=2 \beta\|\operatorname{sen}(\cdot)\|_{L^{2}}^{2}
\end{aligned}
$$

Usando o fato de sen $(\cdot)$ e $\operatorname{sen}^{2}(\cdot)$ não pertencerem a $R\left(D^{2}+1\right)$ somos capazes de provar que $T$ é bijetora. Assim, a primeira conclusão do teorema segue do teorema da função implícita.

A segunda conclusão segue da unicidade dada pelo teorema da função implícita; para isso, é suficiente mostrar que

$$
\left(z^{k}, \beta^{k}, \rho^{k}, \theta^{k}\right) \rightarrow\left(z_{1}, \beta_{1}, \rho_{1}, \theta_{1}\right)=\left(\left(1+i \lim _{k \rightarrow 1} \frac{g_{b}\left(u_{k}\right)}{U_{k}}\right) \xi_{1}, 1,-\lim _{k \rightarrow 1} \frac{g_{b}\left(u_{k}\right)}{U_{k}}, \frac{\pi}{2}\right)
$$

Segue da parte (ii) do teorema (2.1) que $\left\{\rho^{k}\right\}$ e $\left\{\beta^{k}\right\}$ são limitadas e $\left\{\beta^{k}\right.$ sen $(\cdot)+$ $\left.(k-1) z^{k}\right\}$ é limitada em $L^{2}$

Note que $z^{k} \in H^{2} \cap H_{0}^{1} e\left\langle\operatorname{sen}(\cdot), z_{k}\right\rangle=0$, entäo pela parte (iii) do lema (2.1)

$$
3\|z\|_{L^{2}}^{2} \leq\left|\left\langle\left(D^{2}+1\right) z, z\right\rangle\right|
$$

Agora, $h_{1}\left(z^{k}, \beta^{k}, \rho^{k}, \theta^{k}, k\right)=0$, e então:

$$
\frac{1}{3}\left(D^{2}+1\right) z^{k}=\left(\beta^{k} \operatorname{sen}(\cdot)+(k-1) z^{k}\right) \frac{1}{3}\left[1-i \rho^{k}+\frac{k}{(k-1)}\left[g_{a}\left(U_{k}\right)+g_{b}\left(U_{k}\right) e^{-i \theta}\left(\frac{e^{i \omega \delta}-1}{i \omega \delta}\right)\right]\right.
$$

Daí,

$$
\left|z^{k}\right|_{L^{2}}^{2} \leq\left|\left\langle\left(\beta^{k} \operatorname{sen}(\cdot)+(k-1) z^{k}\right) \omega\left(\rho^{k}, \theta^{k}, k\right), z^{k}\right\rangle\right|
$$

onde $\omega\left(\rho^{k}, \theta^{k}, k\right)=\frac{1}{3}\left[1-i \rho^{k}+\frac{k}{(k-1)}\left(g_{a}\left(U_{k}\right)+g_{b}\left(U_{k}\right) e^{-i \theta^{k}}\left(\frac{e^{i \omega \delta}-1}{i \omega \delta}\right)\right)\right]$

Da existência de $\lim _{k \rightarrow 1} \frac{k}{(k-1)}\left(g_{a}\left(U_{k}\right)+g_{b}\left(U_{k}\right) e^{-i \theta^{k}}\left(\frac{e^{i \omega \delta}-1}{i \omega \delta}\right)\right)$, e do fato que $\left\{\rho^{k}\right\}$ é limitada para $k \in\left[1, k^{*}\right]$, implica que existe $M \geq 0$ tal que

$$
\left|\omega\left(\rho^{k}, \theta^{k}, k\right)\right|_{\text {sup }}<M, \quad k \in\left[1, k^{*}\right]
$$


Assim, $\left\{z^{k}\right\}$ é limitada em $L^{2}$.

Como $\left(D^{2}+1\right):\left(H^{2} \cap H_{0}^{1}\right) \cap R\left(D^{2}+1\right) \rightarrow R\left(D^{2}+1\right)$ tem uma inversa limitada, aplicando $\left(D^{2}+1\right)^{-1}$ em $h_{1}\left(z^{k}, \beta^{k}, \rho^{k}, \theta^{k}, k\right)=0$, vemos que $\left\{z^{k}\right\}$ é também limitada em $H^{2} \cap H_{0}^{1}$, e assim, $\left\{\left(z^{k}, \beta^{k}, \rho^{k}, \theta^{k}\right), k \in\left(1, k^{*}\right]\right\}$ é precompacta em $L^{2} \times \mathbb{R}^{3}$.

Seja $\left\{z_{k_{n}}, \beta_{k_{n}}, \rho_{k_{n}}, \theta_{k_{n}}\right\}$ qualquer subsequência tal que:

$$
\left\{z_{k_{n}}, \beta_{k_{n}}, \rho_{k_{n}}, \theta_{k_{n}}\right\} \rightarrow\left(z^{1}, \beta^{1}, \rho^{1}, \theta^{1}\right), k_{n} \rightarrow 1, n \rightarrow \infty
$$

Afirmamos que $\left(z^{1}, \beta^{1}, \rho^{1}, \theta^{1}\right)=\left(z_{1}, \beta_{1}, \rho_{1}, \theta_{1}\right)$.

De fato, de $\lim _{n \rightarrow \infty} H\left(z^{k_{n}}, \beta^{k_{n}}, \rho^{k_{n}}, \theta^{k_{n}}, k_{n}\right)=0$, obtemos:

$$
\begin{gathered}
\left(D^{2}+1\right) z^{1}+\beta^{1} \operatorname{sen}(\cdot)\left[-\alpha_{1}\left(1-\lim _{k \rightarrow 1} \frac{g_{b}\left(u_{k}\right)}{U_{k}} e^{-i \theta^{1}}\right) \operatorname{sen}(\cdot)-i \rho^{1}+1\right]=0 \\
\left\langle\operatorname{sen}(\cdot), z^{1}\right\rangle=0=\left(\left(\beta^{1}\right)^{2}-1\right)\|\operatorname{sen}(\cdot)\|_{L^{2}}^{2}
\end{gathered}
$$

Então, $\beta^{1}=1=\beta_{1}$.

Multiplicando por $\operatorname{sen}(\cdot)$,

$$
\left(D^{2}+1\right) z^{1} \operatorname{sen}(\cdot)+\left(1-i \rho^{1}\right) \operatorname{sen}^{2}(\cdot)-\alpha_{1}\left(1-\lim _{k \rightarrow 1} \frac{g_{b}\left(u_{k}\right)}{U_{k}} e^{-i \theta^{1}}\right) \operatorname{sen}^{3}(\cdot)=0
$$

Integrando de 0 a $\pi$,

$$
\left(1-i \rho^{1}\right) \int_{0}^{\pi} \operatorname{sen}^{2}(x) d x=\alpha_{1}\left(1-\lim _{k \rightarrow 1} \frac{g_{b}\left(u_{k}\right)}{U_{k}} e^{-i \theta^{1}}\right) \int_{0}^{\pi} \operatorname{sen}^{3}(x) d x
$$

Logo, $1-i \rho^{1}=1-\lim _{k \rightarrow 1} \frac{g_{b}\left(u_{k}\right)}{U_{k}} e^{-i \theta^{1}}$, o que implica que $\theta^{1}=\frac{\pi}{2}$ e também, $\rho^{1}=-\lim _{k \rightarrow 1} \frac{g_{b}\left(u_{k}\right)}{U_{k}}$

Assim, 


$$
\begin{gathered}
\left(D^{2}+1\right) z^{1}+\left[1+i \lim _{k \rightarrow 1} \frac{g_{b}\left(u_{k}\right)}{U_{k}}-\alpha_{1}\left(1+i \lim _{k \rightarrow 1} \frac{g_{b}\left(u_{k}\right)}{U_{k}}\right) \operatorname{sen}(\cdot)\right] \operatorname{sen}(\cdot)=0 \\
\left\langle\operatorname{sen}(\cdot), z^{1}\right\rangle=0
\end{gathered}
$$

Como a solução desta equação em $H^{2} \cap H_{0}^{1}$ é única, segue que $z^{1}=z_{1}$.

Como consequência imediata temos:

Corolário 2.3. Se $0<k^{*}-1<<1$, então para cada $k \in\left(1, k^{*}\right)$, o problema do autovalor

$$
\Delta(k, i \omega, \tau) y=0, \quad 0 \neq y \in H^{2} \cap H_{0}^{1}
$$

tem uma solução $(\omega, \tau, y)$, ou equivalentemente, $i \omega \in \sigma\left(A_{\tau}(k)\right)$ se, e somente se,

$$
\begin{gathered}
\omega=\omega_{k}=(k-1) \rho_{k} \\
\tau=\tau_{k_{n}}=\frac{\theta_{k}+2 n \pi}{\omega_{k}}, \quad n=0,1,2, \ldots \\
y=c y_{k}, \quad y_{k}=\beta_{k} \operatorname{sen}(\cdot)+(k-1) z_{k}
\end{gathered}
$$

Aqui, c é qualquer constante não nula, $e z_{k}, \beta_{k}, \rho_{k}, \theta_{k}$ são definidos como no teorema $(2.2)$. 


\section{Capítulo 3}

\section{Estabilidade do Equílibrio Positivo}

Segundo a teoria desenvolvida por Huang em [13], daremos condições que garantem a estabilidade assintótica do equilíbrio positivo $U_{k}$.

Teorema 3.1. Sejam $k>1$ e. $M(k)=\max _{x \in[0, \pi]}\left\{-g_{b}\left(U_{k}(x)\right)\right\}$.

$S e \frac{k M(k)}{\delta} \int_{-\tau}^{-\tau+\delta} s d s>\frac{-\pi}{2}$, então o equilíbrio positivo é localmente assintoticamente estável.

Em [13], Huang descreve a localização das raízes da equação

$$
\Delta(\lambda)=\lambda+a+\int_{0}^{r} e^{-\lambda s} d \eta(s)
$$

através dos seguintes lemas:

Lema 3.2. Huang Para qualquer $\theta \in(0, \pi)$, introduzimos

$$
D_{\theta}=\left\{x+i y: x \in \mathbb{R}, y \geq-\frac{\operatorname{sen} \theta-\theta \cos \theta}{\theta \operatorname{sen} \theta} x\right\},
$$

um semi-plano do plano complexo $\mathbb{C}$. Então para qualquer $z_{i} \in D_{\theta}, \alpha_{i} \geq 0, i=1,2$, nós temos que $\alpha_{1} z_{1}+\alpha_{2} z_{2} \in D_{\theta}$. Além disso,

$$
z_{1}+z_{2}=0 \text { se, e somente se, } z_{1}, z_{2} \in \partial D_{0} \text { e } z_{1}=-z_{2} .
$$


Lema 3.3. Huang Para cada $\theta \in(0, \pi)$, seja $W_{\theta}:[0, \infty) \rightarrow \mathbb{C}$ dado por

$$
\begin{aligned}
W_{\theta}(v) & =-\theta \cos \theta / \operatorname{sen} \theta+i v+\theta e^{-i v} / \operatorname{sen} \theta \\
& -\theta(\cos \theta-\cos v) / \operatorname{sen} \theta+i(v-\theta \operatorname{sen} v / \operatorname{sen} \theta)
\end{aligned}
$$

então $W_{\theta}([0, \infty)) \in D_{\theta}$.

Antes de provarmos o teorema (3.1), estabelecemos o seguinte resultado:

Lema 3.4. Todos os autovalores de $A(k)$ são reais e não-positivos.

Prova: Como $A(k)$ é um operador auto-adjunto, todos os seus autovalores são reais.

Suponha que $A(k)$ tem algum autovalor $\lambda>0$ e seja $y$ a correspondente autofunção. Temos,

$$
D^{2} y+\left[k\left(1+g_{a}\left(U_{k}\right)\right)-\lambda\right] y=0
$$

Porém, $D^{2} U_{k}+k\left(1+\frac{g\left(U_{k}\right)}{U_{k}}\right) U_{k}=0$ e, como por hipótese, numa vizinhança da origem $\frac{g(\xi, \xi)}{\xi}>g_{a}(\xi)$, para $k$ suficientemente próximo de 1 , temos

$$
k\left(1+\frac{g\left(U_{k}\right)}{U_{k}}\right)>k\left(1+g_{a}\left(U_{k}\right)\right)-\lambda
$$

Pelo teorema da comparação de Sturm, $U_{k}$ tem pelo menos um zero entre 0 $e \pi$, o que contradiz o fato de $U_{k}$ ser positivo neste intervalo. Portanto $A(k)$ tem apenas autovalores não-positivos.

Como corolário temos:

Corolário 3.5. Para toda $\psi \in H^{2} \cap H_{0}^{1}$,

$$
\int_{0}^{\pi}(A(k) \psi) \bar{\psi} d x \leq 0
$$

Prova: Como $C_{0}^{2}=\left\{y \in C^{2}((0, \pi)) \cap C([0, \pi]), y(0)=y(\pi)=0\right\}$ é denso em $L^{2}[0, \pi]$, basta considerarmos $\psi \in C_{0}^{2}$. 
Como $A(k)$ é auto-adjunto, a coleção $\{\psi\}$ de todas as autofunções com $\int_{0}^{\pi} \psi^{2} d x=$ 1 formam uma base de $C_{0}^{2}$ ([19], pag. 374). A desigualdade (3.1) segue do lema (3.4) e da equaf̧ão de Parseval.

Prova do teorema (3.1): Lembremos que o problema do aoutovalor consiste em encontrar $\psi \in H^{2} \cap H_{0}^{1}, \psi \neq 0$, e $\lambda \in \mathbb{C}$, tal que

$$
\Delta(k, \lambda, \tau) \psi:=\left[D^{2}+k\left(1+g_{a}\left(U_{k}\right)\right)+\frac{k}{\delta} \int_{-\tau}^{-\tau+\delta} g_{b}\left(U_{k}\right) e^{\lambda s} d s-\lambda\right] \psi=0
$$

Nós afirmamos que a equação característica não tem autovalor $\lambda$ com $\operatorname{Re} \lambda \geq 0$.

Para ver isto, colocamos $\lambda=u+i v$, multiplicamos por $\Delta(k, \lambda, \tau) \psi$ por $\bar{\psi} e$ integramos de 0 a $\pi$, obtendo:

$$
\int_{0}^{\pi} \Delta(k, \lambda, \tau) \psi \bar{\psi} d x=\int_{0}^{\pi}\left[u+i v-\frac{k}{\delta} \int_{-\tau}^{-\tau+\delta} g_{b}\left(U_{k}\right) e^{\lambda s} d s\right]|\psi|^{2} d x-\int_{0}^{\pi}(A(k) \psi) \bar{\psi}
$$

Para $u=v=0$, observando que numa vizinhança da origem, $g_{b}(\xi)<0$,

$$
\begin{aligned}
\int_{0}^{\pi} \Delta(k, 0, \tau) \psi \bar{\psi} d x & =-\frac{k}{\delta} \int_{-\tau}^{-\tau+\delta} d s \int_{0}^{\pi} g_{b}\left(U_{k}\right)|\psi|^{2} d x-\int_{0}^{\pi}(A(k) \psi) \bar{\psi} d x \\
& >-\frac{k}{\delta} \int_{-\tau}^{-\tau+\delta} d s \int_{0}^{\pi} g_{b}\left(U_{k}\right)|\psi|^{2} d x>0
\end{aligned}
$$

Assim, $\Delta(k, 0, \tau) \psi \neq 0$, para toda $\psi \in H^{2} \cap H_{0}^{l}, \psi \neq 0$

Se $u \geq 0, v \geq 0$ e $u+v>0$, então 


$$
\begin{aligned}
\int_{0}^{\pi} \Delta(k, 0, \tau) \psi \bar{\psi} d x= & u \int_{0}^{\pi}|\psi|^{2} d x-\int_{0}^{\pi}(A(k) \psi) \bar{\psi} d x+ \\
& i v \int_{0}^{\pi}\left[1-\frac{2 k}{\delta \pi} g_{b}\left(U_{k}\right) \int_{-\tau}^{-\tau+\delta} e^{u s} s d s\right]|\psi|^{2} d x \\
& -\frac{2 k}{\delta \pi} \int_{-\tau}^{-\tau+\delta} e^{u s}\left(-i v s+\frac{\pi}{2} e^{i v s}\right) d s \int_{0}^{\pi} g_{b}\left(U_{k}\right)|\psi|^{2} d x
\end{aligned}
$$

Se definirmos

$$
\begin{aligned}
z_{2} & =-\frac{2 k}{\delta \pi} \int_{-\tau}^{-\tau+\delta} e^{u s}\left(-i v s+\frac{\pi}{2} e^{i v s}\right) d s \int_{0}^{\pi} g_{b}\left(U_{k}\right)|\psi|^{2} d x \\
& =-\frac{2 k}{\delta \pi} \int_{-\tau}^{-\tau+\delta} e^{u s} W_{\frac{\pi}{2}}(-v s) d s \int_{0}^{\pi} g_{b}\left(U_{k}\right)|\psi|^{2} d x
\end{aligned}
$$

como $W_{\frac{\pi}{2}}(-v s) \in D_{\frac{\pi}{2}}$ e $z_{2}$ é uma combinação infinita de termos positivos multiplicados por $W_{\frac{\pi}{2}}(-v s)$ e $D_{\frac{\pi}{2}}$ é fechado, então $z_{2} \in D_{\frac{\pi}{2}}$.

\section{Temos}

$$
u \int_{0}^{\pi}|\psi|^{2} d x-\int_{0}^{\pi}(A(k) \psi) \bar{\psi} d x>u \int_{0}^{\pi}|\psi|^{2} d x
$$

Por hipótese, $\frac{k M(k)}{\delta} \int_{-\tau}^{-\tau+\delta} s d s>\frac{-\pi}{2}$, daí

$$
1-\frac{2 k}{\delta \pi} g_{b}\left(U_{k}\right) \int_{-\tau}^{-\tau+\delta} e^{u s} s d s \geq 1+\frac{2 k M(k)}{\delta \pi} \int_{-\tau}^{-\tau+\delta} s d s=\sigma>0
$$

Portanto,

$v \int_{0}^{\pi}\left[1-\frac{2 k}{\delta \pi} g_{b}\left(U_{k}\right) \int_{-\tau}^{-\tau+\delta} e^{u s} s d s\right]|\psi|^{2} d x \geq v \sigma \int_{0}^{\pi}|\psi|^{2} d x>0$, se $v>0$

\section{Fazendo}

$$
z_{1}=u \int_{0}^{\pi}|\psi|^{2} d x-\int_{0}^{\pi}(A(k) \psi) \bar{\psi} d x+i v \int_{0}^{\pi}\left[1-\frac{2 k}{\delta \pi} g_{b}\left(U_{k}\right) \int_{-\tau}^{-\tau+\delta} e^{u s} s d s\right]|\psi|^{2} d x
$$


$z_{1} \in D_{\theta}$, para todo $\theta \in(0, \pi)$, em particular para $\theta=\frac{\pi}{2}$.

Porém, $z_{1} \notin \partial D_{\frac{\pi}{2}}$, pois $\operatorname{Re} z_{1} \geq 0$ eIm $z_{1} \geq 0$, mas Re $z_{1}+I m z_{1}>0$.

Logo, $z_{1}+z_{2} \neq 0$. Assim

$$
\int_{0}^{\pi} \Delta(k, \lambda, \tau) \psi \bar{\psi} \neq 0 \text { e } \Delta(k, \lambda, \tau) \psi \neq 0
$$

para todo $u \geq 0, v \geq 0$ e $\psi \in H^{2} \cap H_{0}^{1}, \psi \neq 0$.

Para concluir, notamos que se $u \geq 0$ e $v \leq 0$, para $\psi \in H^{2} \cap H_{0}^{1}, \psi \neq 0$,

$$
\Delta(k, u+i v, \tau) \psi=\bar{\Delta}(k, u-i v, \tau) \bar{\psi} \neq 0
$$

Agora, vamos analisar a estabilidade de $U_{k} \operatorname{com} k \in\left(1, k^{*}\right)$ fixo, c o retardo $\tau$ agora sendo tratado como parâmetro. Para descrever a estabilidade de $U_{k}$, é suficiente investigar como o autovalor $\lambda=i \omega$ varia quando o retardo $\tau$ passa através de cada $\tau_{k_{n}}, n=0,1,2, \ldots$

Lema 3.6. (i) Se $0<k^{*}-1<<1$, então para $k \in\left(1, k^{*}\right]$

$$
\begin{aligned}
S_{k_{n}} & \stackrel{\operatorname{def}}{=} \int_{0}^{\pi}\left[1+\tau_{k_{n}} k e^{-i \theta_{k}} g_{b}\left(U_{k}\right)\left[\frac{e^{i \omega_{k} \delta}-1}{i \omega_{k} \delta}\right]-k g_{b}\left(U_{k}\right) e^{-i \theta_{k}} \frac{e^{i \omega_{k} \delta}}{i \omega_{k}}\right. \\
& \left.-i k g_{b}\left(U_{k}\right) \frac{e^{-i \theta_{k}}}{\omega_{k}}\left[\frac{e^{i \omega_{k} \delta}-1}{i \omega_{k} \delta}\right]\right] y_{k}^{2}(x) d x \neq 0
\end{aligned}
$$

(ii) Para cada $k \in\left(1, k^{*}\right), 0<k^{*}-1<<1$, e $n=0,1,2, \ldots, \lambda=i \omega_{k}$ é um autovalor simples de $A_{\tau_{k_{n}}}(k)$.

$$
\text { (iii) } \lim _{k \rightarrow 1} \bar{S}_{k_{n}}=\left[1-i\left(2 n \pi+\frac{\pi}{2}\right)\right] \int_{0}^{\pi} \operatorname{sen}^{2}(x) d x \text {. }
$$

Prova:(i) Dos resultados da seção anterior temos

$$
\begin{gathered}
y_{k}=\beta_{k} \operatorname{sen}(\cdot)+(k-1) z_{k} \\
U_{k}=\alpha_{k}(k-1) \operatorname{sen}(\cdot)+\alpha_{k}(k-1)^{2} z_{k}
\end{gathered}
$$




$$
\tau_{k_{n}}=\frac{\theta_{k}+2 n \pi}{(k-1) \rho_{k}}
$$

onde $\theta_{k} \rightarrow \frac{\pi}{2}, \rho_{k} \rightarrow-\lim _{k \rightarrow 1} \frac{g_{b}\left(u_{k}\right)}{U_{k}}, \beta_{k} \rightarrow 1, \alpha_{k} \rightarrow \frac{\int_{0}^{\pi} \operatorname{sen}^{2}(x) d x}{\int_{0}^{\pi} \operatorname{sen}^{3}(x) d x}$, quando $k \rightarrow 1$. Seja $t_{k}=\arg \left(\int_{0}^{\pi} y_{k}^{2}(x) d x\right),-\pi<t_{k} \leq \pi ;$ entäo

$$
\left|\int_{0}^{\pi} y_{k}^{2}(x) d x\right| e^{i t_{k}}=\int_{0}^{\pi} y_{k}^{2}(x) d x \stackrel{k \rightarrow 1}{\rightarrow} \int_{0}^{\pi} \operatorname{sen}^{2}(x) d x
$$

Logo, $t_{k} \rightarrow 0$ quando $k \rightarrow 1$. Além disso,

$$
\begin{gathered}
\int_{0}^{\pi}\left[e^{-i t_{k}}+\tau_{k_{n}} k e^{-i\left(\theta_{k}+l_{k}\right)} g_{b}\left(U_{k}\right)\left[\frac{e^{i \omega_{k} \delta}-1}{i \omega_{k} \delta}\right]-k g_{b}\left(U_{k}\right) e^{-i\left(\theta_{k}+t_{k}\right)} \frac{e^{i \omega_{k} \delta}}{i \omega_{k}}+\right. \\
\left.-i k g_{b}\left(U_{k}\right) \frac{e^{-i\left(\theta_{k}+t_{k}\right)}}{\omega_{k}}\left[\frac{e^{i \omega_{k} \delta}-1}{i \omega_{k} \delta}\right]\right] y_{k}^{2}(x) d x= \\
\int_{0}^{\pi} e^{-i t_{k}} y_{k}^{2}(x) d x+\frac{\theta_{k}+2 n \pi}{\rho_{k}} k \int_{0}^{\pi} e^{-i\left(\theta_{k}+t_{k}\right)} \frac{g_{b}\left(U_{k}\right)}{k-1}\left[\frac{e^{i \omega_{k} \delta}-1}{i \omega_{k} \delta}\right] y_{k}^{2}(x) d x+ \\
-k \int_{0}^{\pi} g_{b}\left(U_{k}\right) e^{-i\left(\theta_{k}+t_{k}\right)} \frac{e^{i \omega_{k} \delta}}{i \omega_{k}} y_{k}^{2}(x) d x-i k \int_{0}^{\pi} g_{b}\left(U_{k}\right) \frac{e^{-i\left(\theta_{k}+t_{k}\right)}}{\omega_{k}}\left[\frac{e^{i \omega_{k} \delta}-1}{i \omega_{k} \delta}\right] y_{k}^{2}(x) d x= \\
\left|\int_{0}^{\pi} y_{k}^{2}(x) d x\right|+\frac{\theta_{k}+2 n \pi}{\rho_{k}} k \int_{0}^{\pi} e^{-i\left(\theta_{k}+t_{k}\right)} \frac{g_{b}\left(U_{k}\right)}{U_{k}} \frac{U_{k}}{k-1}\left[\frac{e^{i \omega_{k} \delta}-1}{i \omega_{k} \delta}\right] y_{k}^{2}(x) d x+ \\
-k \int_{0}^{\pi} \frac{g_{b}\left(U_{k}\right)}{U_{k}} \frac{U_{k}}{k-1} e^{-i\left(\theta_{k}+t_{k}\right)} e^{i \omega_{k} \delta} \frac{(k-1)}{i \omega_{k}} y_{k}^{2}(x) d x+ \\
-i k \int_{0}^{\pi} \frac{g_{b}\left(U_{k}\right)}{U-k} \frac{U_{k}}{k-1} e^{-i\left(\theta_{k}+t_{k}\right)} \frac{(k-1)}{\omega_{k}}\left[\frac{e^{i \omega_{k} \delta}-1}{i \omega_{k} \delta}\right] y_{k}^{2}(x) d x=
\end{gathered}
$$




$$
\begin{gathered}
\left|\int_{0}^{\pi} y_{k}^{2}(x) d x\right|+\frac{\theta_{k}+2 n \pi}{\rho_{k}} k \int_{0}^{\pi} e^{-i\left(\theta_{k}+t_{k}\right)} \frac{g_{b}\left(U_{k}\right)}{U_{k}} \alpha_{k}\left(\operatorname{sen}(x)+(k-1) \xi_{k}\right)\left(\beta_{k} \operatorname{sen}(x)+\right. \\
\begin{array}{r}
\left.(k-1) z_{k}\right)^{2}\left[\frac{e^{i \omega_{k} \delta}-1}{i \omega_{k} \delta}\right] d x-k \int_{0}^{\pi} \frac{g_{b}\left(U_{k}\right)}{U_{k}} e^{-i\left(\theta_{k}+t_{k}\right)} e^{i \omega_{k} \delta} \frac{1}{i \rho_{k}} \alpha_{k}\left(\operatorname{sen}(x)+(k-1) \xi_{k}\right) \\
\left(\beta_{k} \operatorname{sen}(x)+(k-1) z_{k}\right)^{2} d x-i k \int_{0}^{\pi} \frac{g_{b}\left(U_{k}\right)}{U_{k}} e^{-i\left(\theta_{k}+t_{k}\right)} \frac{1}{\rho_{k}}\left[\frac{e^{i \omega_{k} \delta}-1}{i \omega_{k} \delta}\right] \alpha_{k}(\operatorname{sen}(x)+ \\
\left.+(k-1) \xi_{k}\right)\left(\beta_{k} \operatorname{sen}(x)+(k-1) z_{k}\right)^{2} d x=
\end{array}
\end{gathered}
$$

Fazendo $k \rightarrow 1$

$$
\begin{aligned}
& \int_{0}^{\pi} \operatorname{sen}(x)^{2}(x) d x+\left(\frac{\pi}{2}+2 n \pi\right) i \int_{0}^{\pi} \alpha_{1} \operatorname{sen}^{3}(x) d x- \\
& \qquad \int_{0}^{\pi} \alpha_{1} \operatorname{sen}^{3}(x)-i^{2} \int_{0}^{\pi} \alpha_{1} \operatorname{sen}^{3}(x) d x= \\
& =\int_{0}^{\pi} \operatorname{sen}(x)^{2}(x) d x+i\left(\frac{\pi}{2}+2 n \pi\right) \int_{0}^{\pi} \operatorname{sen}^{2}(x) d x
\end{aligned}
$$

lembrando que $t_{k} \rightarrow 0, \rho_{k} \rightarrow-\lim _{k \rightarrow 1} \frac{g_{b}\left(u_{k}\right)}{U_{k}} e \lim _{k \rightarrow 1} \frac{e^{i \omega \delta}-1}{i \omega \delta}=1$, quando $k \rightarrow 1$. Aqui, estamos fazendo uso do teorema da convergência dominada para calcular tais limites.

\section{Como}

$$
\begin{aligned}
\lim _{k \rightarrow 1} \int_{0}^{\pi}\left[e^{-i \ell_{k}}+\tau_{k_{n}} k e^{-i\left(\theta_{k}+t_{k}\right)}\right. & g_{b}\left(U_{k}\right)\left[\frac{e^{i \omega_{k} \delta}-1}{i \omega_{k} \delta}\right]-k g_{b}\left(U_{k}\right) e^{-i\left(\theta_{k}+t_{k}\right)} \frac{e^{i \omega_{k} \delta}}{i \omega_{k}} \\
& \left.-i k g_{b}\left(U_{k}\right) \frac{e^{-i\left(\theta_{k}+t_{k}\right)}}{\omega_{k}}\left[\frac{e^{i \omega_{k} \delta}-1}{i \omega_{k} \delta}\right]\right] y_{k}^{2}(x) d x=\lim _{k \rightarrow 1} S_{k_{n}},
\end{aligned}
$$

para $k \in\left(1, k^{*}\right] S_{k_{n}} \neq 0$

(ii) Pelo corolário (2.3), $\operatorname{dim} N\left[A_{\tau_{k_{n}}}(k)-i \omega_{k}\right]=1, n=0,1,2 \ldots$, com $N\left[A_{\tau_{k_{n}}}(k)-i \omega_{k}\right]=\operatorname{span}\left[e^{i \omega_{k} \cdot} y_{k}\right]$. 
Agora, suponha que $\phi \in \operatorname{Dom}\left(A_{\tau_{k_{n}}}\right) \cap \operatorname{Dom}\left(\left[A_{\tau_{k_{n}}}\right]^{2}\right) e\left[A_{\tau_{k_{n}}}(k)-i \omega_{k}\right]^{2} \phi=0$. Segue que

$$
\left[A_{\tau_{k_{n}}}(k)-i \omega_{k}\right] \phi \in N\left(A_{\tau_{k_{n}}}(k)-i \omega_{k}\right)=\operatorname{span}\left[e^{i \omega_{k}(\cdot)} y_{k}\right]
$$

Assim, existe uma constante $c$ tal que

$$
\left[A_{\tau_{k_{n}}}(k)-i \omega_{k}\right] \phi=c e^{i \omega_{k}(\cdot)} y_{k}
$$

ou

$$
\left\{\begin{array}{l}
\dot{\phi}(\theta)=i \omega_{k} \phi(\theta)+c e^{i \omega_{k} \theta} y_{k}, \theta \in\left[-\tau_{k_{n}}, 0\right] \\
\dot{\phi}(0)=A(k) \phi(0)+\frac{k}{\delta} \int_{-\tau}^{-\tau+\delta} g_{b}\left(U_{k}\right) \phi(s) d s
\end{array}\right.
$$

A primeira equação fornece:

$$
\left\{\begin{array}{l}
\phi(\theta)=\phi(0) e^{i \omega_{k} \theta}+c e^{i \omega_{k} \theta} \theta y_{k} \\
\dot{\phi}(0)=i \omega_{k} \phi(0)+c y_{k}
\end{array}\right.
$$

Substituindo um sistema de equações no outro, vem:

$$
\begin{aligned}
& \Delta\left(k, i \omega_{k}, \tau_{k_{n}}\right) \phi(0)=\left(A(k)+k g_{b}\left(U_{k}\right) e^{-i \omega_{k} \tau_{k_{n}}}\left[\frac{e^{i \omega_{k} \delta}-1}{i \omega_{k} \delta}\right]-i \omega_{k}\right) \phi(0)= \\
& A(k) \phi(0)+k g_{b}\left(U_{k}\right) e^{-i \theta_{k}}\left[\frac{e^{i \omega_{k} \delta}-1}{i \omega_{k} \delta}\right] \phi(0)-i \omega_{k} \phi(0)= \\
& \dot{\phi}(0)-\frac{k}{\delta} \int_{-\tau}^{-\tau+\delta} g_{b}\left(U_{k}\right) \phi(s) d s+k g_{b}\left(U_{k}\right) e^{-i \theta_{k}}\left[\frac{e^{i \omega_{k} \delta}-1}{i \omega_{k} \delta}\right] \phi(0)-\dot{\phi}(0)+c y_{k}= \\
& -\frac{k}{\delta} \int_{-\tau}^{-\tau+\delta} g_{b}\left(U_{k}\right)\left[\phi(0) e^{i \omega_{k} s}+c e^{i \omega_{k} s} s y_{k}\right] d s+k g_{b}\left(U_{k}\right) e^{-i \theta_{k}}\left[\frac{e^{i \omega_{k} \delta}-1}{i \omega_{k} \delta}\right] \phi(0)+c y_{k}=
\end{aligned}
$$




$$
\begin{aligned}
& -k g_{b}\left(U_{k}\right) e^{-i \theta_{k}}\left[\frac{e^{i \omega_{k} \delta}-1}{i \omega_{k} \delta}\right] \phi(0)-\frac{k}{\delta} c \int_{-\tau}^{-\tau+\delta} g_{b}\left(U_{k}\right) e^{i \omega_{k} s} s y_{k} d s+ \\
& +k g_{b}\left(U_{k}\right) e^{-i \theta_{k}}\left[\frac{e^{i \omega_{k} \delta}-1}{i \omega_{k} \delta}\right] \phi(0)+c y_{k}= \\
& =c\left[1-\frac{k}{\delta} \int_{-\tau}^{-\tau+\delta} g_{b}\left(U_{k}\right) e^{i \omega_{k} s} s d s\right] y_{k}
\end{aligned}
$$

e portanto, observando que $A(k)$ é autoadjunta, temos:

$$
\begin{aligned}
& 0=\int_{0}^{\pi} \phi(0)\left[\Delta\left(k, i \omega_{k}, \tau_{k_{n}}\right) y_{k}\right](x) d x=\int_{0}^{\pi} y_{k}(x)\left[\Delta\left(k, i \omega_{k}, \tau_{k_{n}}\right) \phi(0)\right](x) d x= \\
& =c \int_{0}^{\pi}\left(1-\frac{k}{\delta} \int_{-\tau}^{-\tau+\delta} g_{b}\left(U_{k}\right) e^{i \omega_{k} s} s d s\right) y_{k}^{2}(x) d x= \\
& =c \int_{0}^{\pi}\left[1+\tau_{k_{n}} k e^{-i \theta_{k}} g_{b}\left(U_{k}\right)\left[\frac{e^{i \omega_{k} \delta}-1}{i \omega_{k} \delta}\right]-k g_{b}\left(U_{k}\right) e^{-i \theta_{k}} \frac{e^{i \omega_{k} \delta}}{i \omega_{k}}+\right. \\
& \left.\quad-i k g_{b}\left(U_{k}\right) \frac{e^{-i \theta_{k}}}{\omega_{k}}\left[\frac{e^{i \omega_{k} \delta}-1}{i \omega_{k} \delta}\right]\right] y_{k}^{2}(x) d x
\end{aligned}
$$

$e$ por (i), $c=0$.

Portanto, $\left(A_{\tau_{k_{n}}}-i \omega_{k}\right) \phi=0$ e $\phi \in N\left(A_{\tau_{k_{n}}}-i \omega_{k}\right)$. Por indução temos,

$$
N\left(\left[A_{\tau_{k_{n}}}-i \omega_{k}\right]^{j}\right)=N\left(A_{\tau_{k_{n}}}-i \omega_{k}\right), j=1,2, \ldots \text { e } n=0,1,2, \ldots
$$

Portanto, $\lambda=i \omega_{k}$ é um autovalor simples de $A_{\tau_{k_{n}}}$ para $n=0,1,2, \ldots$

(iii) Pela demonstração do item (i)

$$
\lim _{k \rightarrow 1} S_{k_{n}}=\int_{0}^{\pi} \operatorname{sen}^{2}(x) d x+i\left(2 n \pi+\frac{\pi}{2}\right) \int_{0}^{\pi} \operatorname{sen}^{2}(x) d x
$$


Logo,

$$
\lim _{k \rightarrow 1} \bar{S}_{k_{n}}=\left[1-i\left(2 n \pi+\frac{\pi}{2}\right)\right] \int_{0}^{\pi} \operatorname{sen}^{2}(x) d x .
$$

Como $\lambda=i \omega_{k}$ é um autovalor simples de $A_{\tau_{k_{n}}}$, usando o teorema da função implícita nós mostramos que existe uma vizinhança $O_{k_{n}} \times C_{k_{n}} \times H_{k_{n}} \subset \mathbb{R} \times \mathbb{C} \times H_{0}^{1} \cap H^{2}$ de $\left(\tau_{k_{n}}, i \omega_{k}, y_{k}\right)$ e uma transformação continuamente diferenciável $(\lambda, y): O_{k_{n}} \rightarrow$ $C_{k_{n}} \times H_{k_{n}}$ tal que, para cada $\tau \in O_{k_{n}}$, o único autovalor de $A_{\tau_{k_{n}}}$ em $C_{k_{n}}$ é $\lambda(\tau) \mathrm{e}$ $\lambda\left(\tau_{k_{n}}\right)=i \omega_{k}, y\left(\tau_{k_{n}}\right)=y_{k}$ e para cada $\tau \in O_{k_{n}}$,

$$
\Delta(k, \lambda(\tau), \tau) y(\tau)=\left[A(k)+k e^{-\lambda(\tau) \tau} g_{b}\left(U_{k}\right)\left[\frac{e^{\lambda(\tau) \delta}-1}{\lambda(\tau) \delta}\right]-\lambda(\tau)\right] y(\tau)=0
$$

Derivando implicitamente em relação a $\tau$ e calculando $\mathrm{em} \tau=\tau_{k_{n}}$, temos:

$$
\begin{aligned}
& \left\{-\frac{d \lambda\left(\tau_{k_{n}}\right)}{d \tau} \tau_{k_{n}} k g_{b}\left(U_{k}\right) e^{-\lambda\left(\tau_{k_{n}}\right) \tau_{k_{n}}}\left[\frac{e^{\lambda\left(\tau_{k_{n}}\right) \delta}-1}{\lambda\left(\tau_{k_{n}}\right) \delta}\right]-\lambda\left(\tau_{k_{n}}\right) k g_{b}\left(U_{k}\right) e^{-\lambda\left(\tau_{k_{n}}\right) \tau_{k_{n}}}\left[\frac{e^{\lambda\left(\tau_{k_{n}}\right) \delta}-1}{\lambda\left(\tau_{k_{n}}\right) \delta}\right]+\right. \\
& \left.+k g_{b}\left(U_{k}\right) e^{-\lambda\left(\tau_{k_{n}}\right) \tau_{k_{n}}}\left[\frac{\frac{d \lambda\left(\tau_{k_{n}}\right)}{d \tau} \delta e^{\lambda\left(\tau_{k_{n}}\right) \delta} \lambda\left(\tau_{k_{n}}\right) \delta-\frac{d \lambda\left(\tau_{k_{n}}\right)}{d_{\tau}} \delta\left(e^{\lambda\left(\tau_{k_{n}}\right) \delta}-1\right)}{\lambda\left(\tau_{k_{n}}\right)^{2} \delta^{2}}\right]-\frac{d \lambda\left(\tau_{k_{n}}\right)}{d \tau}\right\} y\left(\tau_{k_{n}}\right)+ \\
& +\Delta\left(k, \lambda\left(\tau_{k_{n}}\right), \tau_{k_{n}}\right) \frac{d y\left(\tau_{k_{n}}\right)}{d \tau}=0 \\
& \left\{-\frac{d \lambda\left(\tau_{k_{n}}\right)}{d \tau} \tau_{k_{n}} k g_{b}\left(U_{k}\right) e^{-i \theta_{k}}\left[\frac{e^{i \omega_{k} \delta}-1}{i \omega_{k} \delta}\right]-i \omega_{k} k g_{b}\left(U_{k}\right) e^{-i \theta_{k}}\left[\frac{e^{i \omega_{k} \delta}-1}{i \omega_{k} \delta}\right]+\right. \\
& \left.+k g_{b}\left(U_{k}\right) e^{-i \theta_{k}} \frac{d \lambda\left(\tau_{k_{n}}\right)}{d \tau}\left[\frac{\delta^{2} e^{i \omega_{k} \delta} i \omega_{k}-\delta\left(e^{i \omega_{k} \delta}-1\right)}{\left(i \omega_{k}\right)^{2} \delta^{2}}\right]-\frac{d \lambda\left(\tau_{k_{n}}\right)}{d \tau}\right\} y_{k}+ \\
& +\Delta\left(k, \lambda\left(\tau_{k_{n}}\right), \tau_{k_{n}}\right) \frac{d y\left(\tau_{k_{n}}\right)}{d \tau}=0
\end{aligned}
$$




$$
\begin{aligned}
& \frac{d \lambda\left(\tau_{k_{n}}\right)}{d \tau}\left\{-1-\tau_{k_{n}} k g_{b}\left(U_{k}\right) e^{-i \theta_{k}}\left[\frac{e^{i \omega_{k} \delta}-1}{i \omega_{k} \delta}\right]+k g_{b}\left(U_{k}\right) e^{-i \theta_{k}} \frac{e^{i \omega_{k} \delta}}{i \omega_{k}}\right. \\
& \left.-k g_{b}\left(U_{k}\right) \frac{e^{m i \theta_{k}}}{i \omega_{k}}\left[\frac{\left.e^{i \omega_{k} \delta}-1\right)}{i \omega_{k} \delta}\right]\right\} y_{k}-i \omega_{k} k g_{b}\left(U_{k}\right) e^{-i \theta_{k}}\left[\frac{e^{i \omega_{k} \delta}-1}{i \omega_{k} \delta}\right] y_{k}+ \\
& \Delta\left(k, \lambda\left(\tau_{k_{n}}\right), \tau_{k_{n}}\right) \frac{d y\left(\tau_{k_{n}}\right)}{d \tau}=0
\end{aligned}
$$

Tomando produto interno com $y_{k}$, observando que $A(k)$ é auto-adjunta e $\Delta\left(k, i \omega_{k}, \tau_{k_{n}}\right) y_{k}=0$ e lembrando a definição de $S_{k_{n}}$, temos:

$$
\begin{aligned}
& \frac{d \lambda\left(\tau_{k_{n}}\right)}{d \tau}=\frac{-\int_{0}^{\pi} i \omega_{k} k g_{b}\left(U_{k}(x)\right) e^{-i \theta_{k}}\left[\frac{e^{i \omega_{k} \delta}-1}{i \omega_{k} \delta}\right] y_{k}^{2}(x) d x}{S_{k_{n}}} \\
& \frac{d \lambda\left(\tau_{k_{n}}\right)}{d \tau}=\frac{-1}{\left|S_{k_{n}}\right|^{2}}\left\{\int_{0}^{\pi} i \omega_{k} k g_{b}\left(U_{k}(x)\right) e^{i \theta_{k}}\left[\frac{e^{i \omega_{k} \delta}-1}{i \omega_{k} \delta}\right] y_{k}^{2}(x) d x \overline{S_{k_{n}}}\right\} \\
& \frac{d \lambda\left(\tau_{k_{n}}\right)}{d \tau}=\frac{-1}{\left|S_{k_{n}}\right|^{2}}\left\{\int_{0}^{\pi} i \omega_{k} k g_{b}\left(U_{k}(x)\right) e^{-i \theta_{k}}\left[\frac{e^{i \omega_{k} \delta}-1}{i \omega_{k} \delta}\right] y_{k}^{2}(x) d x \overline{\int_{0}^{\pi} y_{k}^{2}(x) d x+}\right. \\
& +\int_{0}^{\pi} i \omega_{k} k g_{b}\left(U_{k}(x)\right) e^{-i \theta_{k}}\left[\frac{e^{i \omega_{k} \delta}-1}{i \omega_{k} \delta}\right] y_{k}^{2}(x) d x \overline{\int_{0}^{\pi} \tau_{k_{n}} k g_{b}\left(U_{k}(x)\right) e^{-i \theta_{k}\left[\frac{e^{i \omega_{k} \delta}-1}{i \omega_{k} \delta}\right] y_{k}^{2}(x) d x}} \\
& -\int_{0}^{\pi} i \omega_{k} k g_{b}\left(U_{k}(x)\right) e^{-i \theta_{k}}\left[\frac{e^{i \omega_{k} \delta}-1}{i \omega_{k} \delta}\right] y_{k}^{2}(x) d x \overline{\int_{0}^{\pi} k g_{b}\left(U_{k}(x)\right) e^{-i \theta_{k}} \frac{e^{i \omega_{k} \delta}}{i \omega_{k}} y_{k}^{2}(x) d x} \\
& -\int_{0}^{\pi} i \omega_{k} k g_{b}\left(U_{k}(x)\right) e^{-i \theta_{k}}\left[\frac{e^{i \omega_{k} \delta}-1}{i \omega_{k} \delta}\right] y_{k}^{2}(x) d x \overline{\left.\int_{0}^{\pi} i k g_{b}\left(U_{k}(x)\right) \frac{e^{-i \theta_{k}}}{\omega_{k}}\left[\frac{e^{i \omega_{k} \delta}-1}{i \omega_{k} \delta}\right] y_{k}^{2}(x) d x\right\}}
\end{aligned}
$$




$$
\begin{aligned}
& \frac{d \lambda\left(\tau_{k_{n}}\right)}{d \tau}=\frac{-1}{\left|S_{k_{n}}\right|^{2}}\left\{\int_{0}^{\pi} i \omega_{k} k g_{b}\left(U_{k}(x)\right) e^{-i \theta_{k}}\left[\frac{e^{i \omega_{k} \delta}-1}{i \omega_{k} \delta}\right] y_{k}^{2}(x) d x\left|\int_{0}^{\pi} y_{k}^{2}(x) d x\right| e^{-i \ell_{k}}+\right. \\
& +i \omega_{k} k^{2} \tau_{k_{n}}\left|\int_{0}^{\pi} g_{b}\left(U_{k}(x)\right)\left[\frac{e^{i \omega_{k} \delta}-1}{i \omega_{k} \delta}\right] y_{k}^{2}(x) d x\right|^{2} \\
& \left.-i \omega_{k} k^{2}\left[\frac{e^{i \omega_{k} \delta}-1}{i \omega_{k} \delta}\right] \frac{e^{-i \omega_{k} \delta}}{\left(-i \omega_{k}\right)}\left|\int_{0}^{\pi} g_{b}\left(U_{k}(x)\right) y_{k}^{2}(x) d x\right|^{2}+k^{2}\left|\int_{0}^{\pi} g_{b}\left(U_{k}(x)\right)\left[\frac{e^{i \omega_{k} \delta}-1}{i \omega_{k} \delta}\right] y_{k}^{2}(x) d x\right|^{2}\right\} \\
& \frac{d \lambda\left(\tau_{k_{n}}\right)}{d \tau}=\frac{-1}{\left|S_{k_{n}}\right|^{2}}\left\{i \omega_{k} k \int_{0}^{\pi} g_{b}\left(U_{k}(x)\right) e^{-i\left(\theta_{k}+t_{k}\right)}\left[\frac{e^{i \omega_{k} \delta}-1}{i \omega_{k} \delta}\right] y_{k}^{2}(x) d x\left|\int_{0}^{\pi} y_{k}^{2}(x) d x\right|+\right. \\
& +i \omega_{k} k^{2} \tau_{k_{n}}\left|\int_{0}^{\pi} g_{b}\left(U_{k}(x)\right)\left[\frac{e^{i \omega_{k} \delta}-1}{i \omega_{k} \delta}\right] y_{k}^{2}(x) d x\right|^{2} \\
& \left.+k^{2}\left[\frac{1-e^{i \omega_{k} \delta}}{i \omega_{k} \delta}\right]\left|\int_{0}^{\pi} g_{b}\left(U_{k}(x)\right) y_{k}^{2}(x) d x\right|^{2}+k^{2}\left[\frac{e^{i \omega_{k} \delta}-1}{i \omega_{k} \delta}\right]^{2}\left|\int_{0}^{\pi} g_{b}\left(U_{k}(x)\right) y_{k}^{2}(x) d x\right|^{2}\right\}
\end{aligned}
$$

Logo,

$$
\begin{aligned}
& \frac{d \lambda\left(\tau_{k_{n}}\right)}{d \tau}=\frac{-1}{\left|S_{k_{n}}\right|^{2}}\left\{i \omega_{k} k \int_{0}^{\pi} g_{b}\left(U_{k}(x)\right) e^{-i\left(\theta_{k}+t_{k}\right)}\left[\frac{e^{i \omega_{k} \delta}-1}{i \omega_{k} \delta}\right] y_{k}^{2}(x) d x\left|\int_{0}^{\pi} y_{k}^{2}(x) d x\right|+\right. \\
& \left.+k^{2}\left[\frac{1-e^{i \omega_{k} \delta}}{i \omega_{k} \delta}\right]\left|\int_{0}^{\pi} g_{b}\left(U_{k}(x)\right) y_{k}^{2}(x) d x\right|^{2}+k^{2}\left[\frac{e^{i \omega_{k} \delta}-1}{i \omega_{k} \delta}\right]^{2}\left|\int_{0}^{\pi} g_{b}\left(U_{k}(x)\right) y_{k}^{2}(x) d x\right|^{2}\right\}
\end{aligned}
$$

Assim, para cada $k \in\left(1, k^{*}\right]$ :

$$
\begin{aligned}
& \frac{d \lambda\left(\tau_{k_{n}}\right)}{d \tau} \equiv \frac{1}{\left|S_{k_{n}}\right|^{2}} \omega_{k} k \rho_{1}\left|\int_{0}^{\pi} \operatorname{sen}^{2}(x) d x\right|^{2}>0 \\
& \text { Portanto } \operatorname{Re} \frac{d \lambda\left(\tau_{k_{n}}\right)}{d \tau}>0 .
\end{aligned}
$$


Com o resultado acima e o teorema (3.1) podemos, então, descrever o equilíbrio positivo do sistema (1) da seguinte forma:

Teorema 3.7. Para cada $0<k-1 \ll 1$, o gerador infinitesimal $A_{\tau}(k)$ tem exatamente $2(n+1)$ autovalores com parte real positiva se $\tau \in\left(\tau_{k_{n}}, \tau_{k_{n}+1}\right], n=0,1,2, \ldots$, e todos os autovalores de $A_{\tau}(k)$ têm parte real negativa se $0 \leq \tau<\tau_{k_{0}}$.

Mais ainda, o equilibrio positivo $U_{k}$ é localmente assintoticamente estável se $0 \leq \tau<\tau_{k_{0}} ;$ e instável se $\tau>\tau_{k_{0}}$ e $\frac{k M(k)}{\delta} \int_{-\tau_{k_{0}}}^{-\tau_{k_{0}}+\delta} s d s \leq \frac{-\pi}{2}$. 


\section{Capítulo 4}

\section{Bifurcação de Hopf}

Fixamos $k \in\left(1, k^{*}\right)$ e $\tau_{k_{n}}$. Sejam $u(t)=U(t, \cdot)-U_{k}(\cdot)$ e $\alpha=\tau-\tau_{k_{n}}$. Nesta seçāo, vamos considerar $g(a, b)=-a b-a^{3} b^{3}$, para conseguirmos informações mais precisas.

Nós obtemos, assim, uma equaçāo equivalente a equação (1):

$$
\begin{aligned}
\dot{u}(t) & =A(k) u(t)-\frac{k}{\delta}\left(U_{k}+3 U_{k}^{5}\right) \int_{-\alpha-\tau_{k_{n}}}^{-\alpha-\tau_{k_{n}}+\delta} u(t+s) d s \\
& -\frac{k}{\delta}\left(U_{k}+3 U_{k}^{5}\right) \int_{-\alpha-\tau_{k_{n}}}^{\alpha-\tau_{k_{n}}+\delta}\left[u(t) u(t+s)+\sum_{p, p^{\prime}}^{2}\left(\begin{array}{c}
3 \\
p
\end{array}\right)\left(\begin{array}{c}
3 \\
p^{\prime}
\end{array}\right) U_{k}^{p+p^{\prime}} u^{3-p}(t) u^{3-p^{\prime}}(t+s)\right. \\
& \left.+u^{3}(t+s) U_{k}^{3}+3 u^{2}(t+s) U_{k}^{4}\right] d s-k\left[u^{3}(l) U_{k}^{3}+3 u^{2}(t) U_{k}^{4}\right]
\end{aligned}
$$

Scjam $\beta \in[-1,1]$ e $v_{k}=2 \pi / \omega_{k}$ e façamos a seguinte mudança de escala no tempo: $w(t)=u(t(1+\beta))$. Temos, então, que $u(t)$ é uma solução $v_{k}(1+\beta)$-pcriódica de (4.1) se, e somente sc, $w(t)$ é uma solução $v_{k}$-periódica da equação

$$
\dot{w}(t)=A(k) w(t)-\frac{k}{\delta}\left(U_{k}+3 U_{k}^{5}\right) \int_{-\tau_{k_{n}}}^{-\tau_{k_{n}}+\delta} w(t+s) d s+G\left(\alpha, \beta, w_{t}\right)
$$

onde 


$$
\begin{aligned}
G\left(\alpha, \beta, w_{t}\right) & =\beta A(k) w(t)+\frac{k}{\delta}\left(U_{k}+3 U_{k}^{5}\right) \int_{-\tau_{k_{n}}}^{-\tau_{k_{n}}+\delta} w(t+s) d s \\
& -(1+\beta) \frac{k}{\delta} \int_{-\alpha-\tau_{k_{n}}}^{-\alpha \cdot \tau_{k_{n}}+\delta}\left[\left(U_{k}+3 U_{k}^{5}\right) w\left(t+\frac{s}{1+\beta}\right)+w(t) w\left(t+\frac{s}{1+\beta}\right)+\right. \\
& \sum_{p, p^{\prime}}^{2}\left(\begin{array}{c}
3 \\
p
\end{array}\right)\left(\begin{array}{c}
3 \\
p^{\prime}
\end{array}\right) U_{k}^{p+p^{\prime}} w^{3-p}(t) w^{3-p^{\prime}}\left(t+\frac{s}{1+\beta}\right)+w^{3}\left(t+\frac{s}{1+\beta}\right) U_{k}^{3}+ \\
& \left.3 w^{2}\left(t+\frac{s}{1+\beta}\right) U_{k}^{4}\right] d s-(1+\beta) k\left[w^{3}(t) U_{k}^{3}+3 w^{2}(t) U_{k}^{4}\right]
\end{aligned}
$$

Nós estamos olhando para a equação (4.2) como uma perturbação da equação linear

$$
\dot{w}(t)=A(k) w(t)-\frac{k}{\delta}\left(U_{k}+3 U_{k}^{5}\right) \int_{-\tau_{k_{n}}}^{-\tau_{k_{n}}+\delta} w(t+s) d s
$$

Descreveremos uma notação que será usada durante o restante do trabalho.

(i) $\langle y, z\rangle^{*}=\int_{0}^{\pi} y(x) z(x) d x$, para $y, z \in X=L^{2}[0, \pi]$

(ii) Para $\phi \in C=C\left(\left[-\tau_{k_{n}}, 0\right] ; X\right) ; \psi \in C^{*}\left(\left[0, \tau_{k_{n}}\right] ; X\right)$, definimos a seguinte forma bilinear:

$$
\begin{aligned}
(\psi, \phi) & =\langle\psi(0), \phi(0)\rangle^{*}-\int_{-\tau_{k_{n}}}^{-\tau_{k_{n}}+\delta}\left\langle\int_{\tau_{k_{n}}}^{\theta} \frac{k}{\delta} g_{b}\left(U_{k}\right) \psi(\theta-\tau) d \tau, \phi(\theta)\right\rangle^{*} d \theta+ \\
& +\int_{-\tau_{k_{n}}+\delta}^{0}\left\langle\int_{-\tau_{k_{n}}}^{-\tau_{k_{n}}+\delta} \frac{k}{\delta} g_{b}\left(U_{k}\right) \psi\left(-\tau_{k_{n}}+\delta-\tau\right) d \tau, \phi\left(-\tau_{k_{n}}+\delta\right)\right\rangle^{*} d \theta
\end{aligned}
$$

Com relação a esta forma bilinear, o operador $A^{*}$ satisfaz $(\psi, A \phi)=\left(A^{*} \psi, \phi\right)$.

(iii) Sejam $y_{k}$ e $S_{k_{n}}$ definidos como no corolário (2.3) e no lema (3.6), respectivamente, e sejam

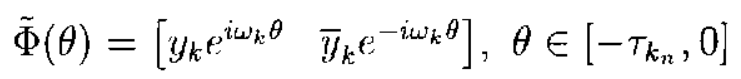




$$
\begin{aligned}
& \bar{\Psi}(s)=\left[\begin{array}{c}
\frac{1}{S_{k_{p}}} y_{k} e^{-i \omega_{k} s} \\
\frac{1}{\bar{S}_{k_{n}}} \bar{y}_{k} e^{i \omega_{k} s}
\end{array}\right], s \in\left[0, \tau_{k_{n}}\right]
\end{aligned}
$$

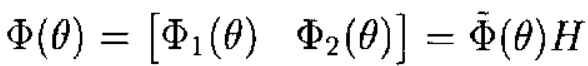

$$
\begin{aligned}
& \Psi(s)=\left[\begin{array}{l}
\Psi_{1}(s) \\
\Psi_{2}(s)
\end{array}\right]=H^{-1} \bar{\Psi}(s)
\end{aligned}
$$

onde $H=\frac{1}{2}\left[\begin{array}{cc}1 & -i \\ 1 & i\end{array}\right]$

(iv) Seja $\Lambda$ o espaço de autofunções de $A_{\tau_{k_{n}}}(k)$ correspondente ao autovalor $\lambda= \pm i \omega_{k}$

Com as notações acima, $\Phi$ é uma base real de $\Lambda$, e $\Psi$ é uma base real do espaço das autofunções da adjunta formal $A_{\tau_{k_{n}}}^{*}(k)$ de $A_{\tau_{k_{n}}}(k)$, correspondente a $\lambda= \pm i \omega_{k}$.

Além disso, usando o fato que $(\psi, A \phi)=\left(A^{*} \psi, \phi\right)$, temos que

$$
\begin{gathered}
i \omega_{k}\left(y_{k} e^{-i \omega_{k^{\prime}}}, \bar{y}_{k} e^{-i \omega_{k^{\prime}}}\right)=\left(A_{\tau_{k_{n}}}^{*} y_{k} e^{-i \omega_{k^{\prime}}}, \bar{y}_{k} e^{-i \omega_{k^{*}}}\right)=\left(y_{k} e^{-i \omega_{k} \cdot}, A_{\tau_{k_{n}}} \bar{y}_{k} e^{-i \omega_{k^{*}}}\right)= \\
=\left(y_{k} e^{-i \omega_{k^{\prime}}},-i \omega_{k} \bar{y}_{k} e^{-i \omega_{k^{*}}}\right)=-i \omega_{k}\left(y_{k} e^{-i \omega_{k}}, \bar{y}_{k} e^{-i \omega_{k^{*}}}\right)
\end{gathered}
$$

e, consequentemente, $\left(y_{k} e^{-i \omega_{k}}, \bar{y}_{k} e^{-i \omega_{k}}\right)=0$

Esta observação nos diz que

$$
\begin{aligned}
& \left\langle y_{k}, \bar{y}_{k}\right\rangle^{*}+\int_{-\tau_{k_{n}}}^{-\tau_{k_{n}}+\delta}\left\langle\int_{-\tau_{k_{n}}}^{\theta} \frac{k}{\delta} g_{b}\left(U_{k}\right) y_{k} e^{-i \omega_{k}(\theta-\tau)} d \tau, \bar{y}_{k} e^{-i \omega_{k} \theta}\right\rangle^{*} d \theta+ \\
& \int_{-\tau_{k_{n}}+\delta}^{0}\left\langle\int_{-\tau_{k_{n}}}^{-\tau_{k_{n}}+\delta} \frac{k}{\delta} g_{b}\left(U_{k}\right) y_{k} e^{-i \omega_{k}\left(-\tau_{k_{n}}+\delta-\tau\right)} d \tau, \bar{y}_{k} e^{-i \omega_{k}\left(-\tau_{k_{n}}+\delta\right)}\right\rangle^{*} d \theta=0
\end{aligned}
$$

isto é, 


$$
\begin{aligned}
& \int_{0}^{\pi} y_{k} \bar{y}_{k} d x+\int_{-\tau_{k_{n}}}^{-\tau_{k_{n}}+\delta} \int_{0}^{\pi} \int_{-\tau_{k_{n}}}^{\theta} \frac{k}{\delta} g_{b}\left(U_{k}\right) y_{k} e^{\left(-2 i \omega_{k} \theta+i \omega_{k} \tau\right)} d \tau \bar{y}_{k} d x d \theta+ \\
& \left(\tau_{k_{n}}-\delta\right) \int_{0}^{\pi} \int_{-\tau_{k_{n}}}^{-\tau_{k_{n}}+\delta} \frac{k}{\delta} g_{b}\left(U_{k}\right) y_{k} e^{-2 i \omega_{k}\left(-\tau_{k_{n}}+\delta\right)} e^{i \omega_{k} \tau} d \tau \bar{y}_{k} d x=0
\end{aligned}
$$

Assim,

$$
\begin{aligned}
& \int_{0}^{\pi} y_{k} \bar{y}_{k} d x+\int_{0}^{\pi} \int_{-\tau_{k_{n}}}^{-\tau_{k_{n}}+\delta} \frac{k}{\delta} g_{b}\left(U_{k}\right) \frac{e^{i \omega_{k} \theta}}{i \omega_{k}} e^{-2 i \omega_{k} \theta} d \theta y_{k} \bar{y}_{k} d x+ \\
& +\int_{0}^{\pi} \int_{-\tau_{k_{n}}}^{-\tau_{k_{n}}+\delta} k g_{b}\left(U_{k}\right) \frac{e^{-i \omega_{k}}}{i \omega_{k}} e^{-2 i \omega_{k} \theta} d \theta y_{k} \bar{y}_{k} d x+ \\
& +\tau_{k_{n}} \int_{0}^{\pi} \int_{-\tau_{k_{n}}}^{-\tau_{k_{n}}+\delta} \frac{k}{\delta} g_{b}\left(U_{k}\right) e^{i \omega_{k} \tau} e^{2 i \theta_{k}} e^{-2 i \omega_{k} \delta} d \tau y_{k} \bar{y}_{k} d x+ \\
& -\int_{0}^{\pi} \int_{-\tau_{k_{n}}}^{-\tau_{k_{n}}+\delta} k g_{b}\left(U_{k}\right) e^{i \omega_{k} \tau} e^{2 i \theta_{k}} e^{-2 i \omega_{k} \delta} d \tau y_{k} \bar{y}_{k} d x=0
\end{aligned}
$$

Com isto, podemos verificar que $(\Psi, \Phi)=I$, onde $I \in \mathbb{R}^{2 \times 2}$ é a identidade.

De fato,

$$
\begin{aligned}
& (\Psi, \Phi)=\langle\Psi(0), \Phi(0)\rangle^{*}+\int_{-\tau_{k_{n}}}^{-\tau_{k_{n}}+\delta}\left\langle\int_{-\tau_{k_{n}}}^{\theta} \frac{k}{\delta} g_{b}\left(U_{k}\right) \Psi(\theta-\tau) d \tau, \Phi(\theta)\right\rangle^{*} d \theta+ \\
& +\int_{-\tau_{k_{n}}+\delta}^{0}\left\langle\int_{-\tau_{k_{n}}}^{-\tau_{k_{n}}+\delta} \frac{k}{\delta} g_{b}\left(U_{k}\right) \Psi\left(-\tau_{k_{n}}+\delta-\tau\right) d \tau, \Phi\left(-\tau_{k_{n}}+\delta\right)\right\rangle^{*} d \theta=
\end{aligned}
$$

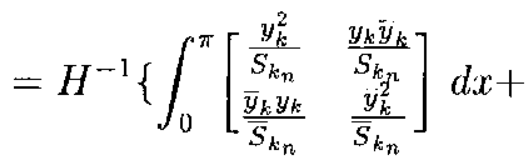

$$
\begin{aligned}
& \int_{0}^{\pi} \int_{=\tau_{k_{n}}}^{-\tau_{k_{n}}+\delta} \int_{-\tau_{k_{n}}}^{\theta} \frac{k}{\delta} g_{b}\left(U_{k}\right)\left[\begin{array}{cc}
\frac{y_{k}^{2} e^{i \omega_{k} \tau}}{S_{k_{n}}} & \frac{y_{k} \ddot{y}_{k} e^{-2 i \omega_{k} \theta} e^{i \omega_{k} \tau}}{S_{k_{n}}} \\
\frac{\bar{y}_{k} y_{k} e^{2 i \omega_{k} e^{-i \omega_{k} \tau}}}{\bar{S}_{k_{n}}} & \frac{\bar{y}_{k}^{2} e^{-i \omega_{k} \tau}}{\bar{S}_{k_{n}}}
\end{array}\right] d \tau d \theta d x+ \\
& \left.\int_{0}^{\pi} \int_{-\tau k_{n}+\delta}^{0} \int_{-\tau_{k_{n}}}^{-\tau_{k_{n}}+\delta} \frac{k}{\delta} g_{b}\left(U_{k}\right)\left[\begin{array}{cc}
\frac{y_{k}^{2} e^{i \omega_{k} \tau}}{S_{k_{n}}} & \frac{y_{k} \bar{y}_{k} e^{-2 i \omega_{k}\left(-\tau_{k_{n}}+\delta\right)} e^{i \omega_{k} \tau}}{S_{k_{n}}} \\
\frac{\bar{y}_{k} y_{k} e^{2 i \omega_{k}\left(-\tau_{k_{n}}+\delta\right)} e^{-i \omega_{k} \tau}}{\bar{S}_{k_{n}}} & \frac{\bar{y}_{k}^{2} e^{-i \omega_{k} \tau}}{\bar{S}_{k_{n}}}
\end{array}\right] d \tau d \theta d x\right\} H
\end{aligned}
$$

Resolvendo todas estas integrais, temos que a segunda entrada é exatamente o termo obtido na observação acima, que é nulo, e a terceira cntrada é o conjugado 
deste termo, portanto, também nulo. Agora, o numerador da primeira entrada é exatamente $S_{k_{n}}$ e o numerador da quarta entrada ć cxatamente $\bar{S}_{k_{n}}$. Obtemos, então, a seguinte conclusão:

$$
(\Psi, \Phi)=H^{-1}\left\{\left[\begin{array}{cc}
S_{k_{n}} & 0 \\
\frac{S_{k_{n}}}{0} & \frac{\dddot{S}_{k_{n}}}{\bar{S}_{k_{n}}}
\end{array}\right]\right\} H=I_{2 \times 2}
$$

Para $\phi \in \mathcal{C}$ a projeção $\pi_{\Lambda}$ de $\phi$ sobre $\Lambda$ é dado por $\pi_{\Lambda}(\phi)=\Phi(\Psi, \phi)$, isto é, $\pi_{\Lambda}(\phi)=\left(\Psi_{1}, \phi\right) \Phi_{1}+\left(\Psi_{2}, \phi\right) \Phi_{2}$.

(v) Seja $\mathcal{P}_{v_{k}}=\left\{f \in \mathcal{C}(\mathbb{R}, X) ; f\left(t+v_{k}\right)=f(t), t \in \mathbb{R}\right\}$ com a norma $\|\cdot\|_{\mathcal{P}_{v_{k}}}$ definida por

$$
\|f\| p_{v_{v_{k}}}=\sup _{t \in\left[0, v_{k}\right]}\|f(t)\|_{X}
$$

para $f \in \mathcal{P}_{v_{k}}$.

(vi) Seja I : $\mathcal{P}_{v_{k}} \rightarrow \mathbb{R}^{2}$ definido por

$$
\mathbf{I} f=\int_{0}^{v_{k}}\langle\Psi(s), f(s)\rangle^{*} d s
$$

onde $\langle\Psi, f\rangle^{*}=\left[\begin{array}{c}\left\langle\Psi_{1}, f\right\rangle^{*} \\ \left\langle\Psi_{2}, f\right\rangle^{*}\end{array}\right]$

Sabemos por [4] que, para cada $f \in \mathcal{P}_{v_{k}}$, a equação

$$
\dot{w}(t)=A(k) w(t)-\frac{k}{\delta} \int_{-\tau_{k_{n}}}^{-\tau_{k_{n}}+\delta}\left(U_{k}+3 U_{k}^{5}\right) w(t+s) d s+f(t)
$$

tem uma solução $v_{k}$-periódica se, e somente se, $f \in N(\mathrm{I})$

Além disso, existc uma projeção contínua $\mathcal{J}: \mathcal{P}_{v_{k}} \rightarrow \mathcal{P}_{v_{k}}$ tal que o conjunto das $f$ em $\mathcal{P}_{v_{k}}$, satisfazendo (4.3) é $(I-\mathcal{J}) \mathcal{P}_{v_{k}}$ e existe um operador linear contínuo $\mathcal{K}:(I-\mathcal{J}) \mathcal{P}_{v_{k}} \rightarrow\left(I-\pi_{\Lambda}\right) \mathcal{P}_{v_{k}}$ tal que $\mathcal{K} f$ é uma solução de (4.4) para cada $f \in(I-\mathcal{J}) \mathcal{P}_{v_{k}}$. Isto é equivalente dizer que $\mathbf{I} f=0$ se, e somente se, $\mathcal{J} f=0$; $\mathcal{K}: N(\mathbf{I}) \rightarrow \mathcal{P}_{v_{k}}$ e $\mathcal{K} f$ é solução de (4.1) se $\pi_{\Lambda}(\mathcal{K} f)=0$ ou $(\Psi, \mathcal{K}(f))=0$. 
Assim, a menos de uma translação, a equação (4.2) tem uma solıção $v_{k^{-}}$ periódica $w(t)$ se, e somente se, existe uma constante $c$ tal que

$$
\left\{\begin{array}{l}
(a) \mathbf{I} G(\alpha, \beta, w)=0 \\
(b) w(t)=c \phi_{1}(t)+\mathcal{K} G(\alpha, \beta, w)(t) ; t \in \mathbb{R}
\end{array}\right.
$$

onde $\Phi_{1}(t)=\frac{1}{2}\left[y_{k} e^{i \omega_{k} t}+\overline{y_{k}} e^{-i \omega_{k} t}\right]=\operatorname{Re}\left(y_{k} e^{i \omega_{k} t}\right), \quad l \in \mathbb{R}$

Nós podemos aplicar o teorema da função implícita para resolver

$$
w(t)=c \phi_{1}(t)+\mathcal{K}(I-\mathcal{J}) G(\alpha, \beta, w)(t) ; t \in \mathbb{R}
$$

para $w^{*}=w^{*}(c, \alpha, \beta)$ e $c, \alpha, \beta$ numa vizinhança do zcro suficientemente pequena, $w^{*}(c, 0,0)-c \Phi_{1}=o(|c|)$ quando $|c| \rightarrow 0$. Também podemos verificar que a função $w^{*}(c, \alpha, \beta)(t)$ é continuamente diferenciável em $c, \alpha, \beta$ e $t$ [veja 14]. Como $G(0,0,0)=0$ e $\frac{\partial G(0,0,0)}{\partial \omega}=0$, temos que $G(0,0, w)=O\left(\|\omega\|^{2}\right)$, e assim,

$$
w^{*}(c, 0,0)-c \Phi_{1}=\mathcal{K}(I-\mathcal{J}) G\left(0,0, w^{*}(c, 0,0)\right)=O\left(\|\omega\|^{2}\right), \text { quando }\|\omega\| \rightarrow 0
$$

Juntando as informações, vemos que $w^{*}(c, 0,0)-c \Phi_{1}=o\left(|c|^{2}\right)$, quando $|c| \rightarrow 0$.

Para cada $c$ sejam $\mu$ e $\gamma$ tais que $\alpha=c \mu$ e $\beta=c \gamma$. Se nós fizermos $w^{*}(c, c \mu, c \gamma)(t)-c \Phi_{1}(t)=c^{2} W(c, t)$, nós temos que $W(c, \cdot) \in \mathcal{P}_{v_{k}}$. Assim, as duas equações em (4.5) são equivalentes a

$$
\left\{\begin{array}{l}
\mathfrak{F}(c, \mu, \gamma, W)=\int_{0}^{v_{k}}\left\langle\Psi, N\left(c, \mu, \gamma, W_{s}\right)\right\rangle^{*} d s=0 \\
W=\mathcal{K} N(c, \mu, \gamma, W)
\end{array}\right.
$$

onde 


$$
\begin{aligned}
& G\left(c \mu, c \gamma, w_{t}\right)=c^{2}\left\{\gamma A(k)\left[\Phi_{1}(t)+c W(c, t)\right]+\frac{k}{\delta} \int_{-\tau_{k_{n}}}^{-\tau_{k_{n}}+\delta}\left(U_{k}+3 U_{k}^{5}\right) W(c, t+s) d s\right. \\
& -(1+c \gamma) \frac{k}{\delta} \int_{-c \mu-\tau_{k_{n}}}^{-c \mu-\tau_{k_{n}}+\delta}\left(U_{k}+3 U_{k}^{5}\right) W\left(c, t+\frac{s}{1+c \gamma}\right) d s \\
& -\gamma \frac{k}{\delta} \int_{-c \mu-\tau_{k_{n}}}^{-c \mu-\tau_{k_{n}}+\delta}\left(U_{k}+3 U_{k}^{5}\right) \Phi_{1}\left(t+\frac{s}{1+c \gamma}\right) d s \\
& +\frac{k}{\delta} \int_{0}^{1} \int_{-\tau_{k_{n}}}^{-\tau_{k_{n}}+\delta}\left(U_{k}+3 U_{k}^{5}\right) \dot{\Phi_{1}}\left(t+s-\theta \frac{c \mu+s c \gamma}{1+c \gamma}\right)\left(\frac{\mu+s \gamma}{1+c \gamma}\right) d s d \theta \\
& -(1+c \gamma) \frac{k}{\delta} \int_{-r . \mu-\tau_{k_{n}}}^{-c \mu-\tau_{k_{n}}+\delta}\left[\Phi_{1}(t)+c W(c, t)\right]\left[\Phi_{1}\left(t+\frac{s}{1+c \gamma}\right)\right. \\
& \left.+c W\left(c, t+\frac{s}{1+c \gamma}\right)\right] d s \\
& -(1+c \gamma) \frac{k}{\delta} \int_{-c \mu-\tau_{k_{n}}}^{-c \mu-\tau_{k_{n}}+\delta} \sum_{p, p^{\prime}}^{2}\left(\begin{array}{l}
3 \\
p
\end{array}\right)\left(\begin{array}{c}
3 \\
p^{\prime}
\end{array}\right) U_{k}^{p+p^{\prime}} c^{4-p-p^{\prime}}\left[\Phi_{1}(t)\right. \\
& +c W(c, t)]^{3-p}\left[\Phi_{1}\left(t+\frac{s}{1+c \gamma}\right)+c W\left(c, t+\frac{s}{1+c \gamma}\right)\right]^{3-p^{\prime}} d s \\
& -(1+c \gamma) \frac{k}{\delta} \int_{-c \mu-\tau_{k_{n}}}^{-c \mu-\tau_{k_{n}}+\delta} U_{k}^{3} c\left[\Phi_{1}\left(t+\frac{s}{1+c \gamma}\right)+c W\left(c, t+\frac{s}{1+c \gamma}\right)\right]^{3} d s \\
& -(1+c \gamma) \frac{k}{\delta} \int_{-c \mu-\tau_{k_{n}}}^{-c \mu-\tau_{k_{n}}+\delta} 3 U_{k}^{4}\left[\Phi_{1}\left(t+\frac{s}{1+c \gamma}\right)+c W\left(c, t+\frac{s}{1+c \gamma}\right)\right]^{2} d s \\
& -(1+c \gamma) k U_{k}^{3} c\left[\Phi_{1}(t)+c W(c, t)\right]^{3} \\
& -(1+c \gamma) 3 k U_{k}^{4}\left[\Phi_{1}(t)+c W(c, t)\right]^{2}=c^{2} N\left(c, \mu, \gamma, W_{t}\right)
\end{aligned}
$$

Como uma solução periódica da equação (4.2) é uma função $C^{1}$, em (4.6), podemos nos restringir a $W \in \mathcal{P}_{v_{k}}^{1}$, onde

$$
\mathcal{P}_{v_{k}}^{1}=\left\{f \in \mathcal{P}_{v_{k}} ; \dot{f} \in \mathcal{P}_{v_{k}}\right\} ; \text { com a norma }\|f\|_{\mathcal{P}_{v_{k}}^{1}}=\|f\|_{\mathcal{P}_{v_{k}}}+\|\dot{f}\|_{\mathcal{P}_{v_{k}}}
$$

Assim,não é difícil ver que $\mathfrak{F}: I_{\bar{\rho}} \times \mathbb{R} \times I_{\bar{\rho}} \times \mathcal{P}_{v_{k}}^{1} \rightarrow \mathbb{R}^{2}$ é continuamente difcrenciável, onde $I_{\bar{\rho}}=[-\bar{\rho}, \bar{\rho}]$; $\operatorname{com} 0<\bar{\rho}<1$.

Como $c^{2} W(c, \cdot)=o(|c|)$, vemos que

$N(0,0,0, W)=-\frac{k}{\delta} \int_{-\tau_{k_{n}}}^{-\tau_{k_{n}}+\delta}\left[1+9 U_{k}^{4}\right] \Phi_{1}(t) \Phi_{1}(t+s) d s-\frac{k}{\delta} \int_{-\tau_{k_{n}}}^{-\tau_{k_{n}}+\delta} 3 U_{k}^{4}\left[\Phi_{1}^{2}(t+s)+\Phi_{1}^{2}(t)\right] d s$ 
Assim,

$$
\begin{aligned}
\mathfrak{F}(0,0,0, W) & =-\int_{0}^{\eta_{k}}\left\langle\Psi(s), \frac{k}{\delta} \int_{-\tau_{k_{n}}}^{-\tau_{k_{n}}+\delta}\left\{\left[1+9 U_{k}^{4}\right] \Phi_{1}(s) \Phi_{1}(s+\theta)\right.\right. \\
& \left.\left.+3 U_{k}^{4}\left[\Phi_{1}^{2}(s+\theta)+\Phi_{1}^{2}(s)\right]\right\} d \theta\right\rangle^{*} d s
\end{aligned}
$$

Usando as definições de $\Psi, \Phi_{1}$, temos:

$$
\mathfrak{F}(0,0,0, W)=\frac{-k}{\delta} H^{-1} \int_{0}^{v_{k}} \int_{0}^{\pi}\left[\begin{array}{ll}
a_{11} & a_{12} \\
a_{21} & a_{22}
\end{array}\right] d x d s H
$$

com,

$$
\begin{aligned}
a_{11} & =\frac{\left(1+9 U_{k}^{4}\right)}{4 S_{k_{n}}}\left[y_{k}^{3} e^{i \omega_{k} s} \int_{-\tau_{k_{n}}}^{-\tau_{k_{n}}+\delta} e^{i \omega_{k} \theta} d \theta+y_{k}^{2} \bar{y}_{k} e^{-i \omega_{k} s} \int_{-\tau_{k_{n}}}^{-\tau_{k_{n}}+\delta} e^{-i \omega_{k} \theta} d \theta\right. \\
& \left.+\bar{y}_{k} y_{k}^{2} e^{-i \omega_{k} s} \int_{-\tau_{k_{n}}}^{-\tau_{k_{n}}+\delta} e^{i \omega_{k} \theta} d \theta+y_{k} \bar{y}_{k}^{2} e^{-3 i \omega_{k} s} \int_{-\tau_{k_{n}}}^{-\tau_{k_{n}}+\delta} e^{-i \omega_{k} \theta} d \theta\right] \\
a_{12} & =\frac{\left(3 U_{k}^{4}\right)}{4 S_{k_{n}}}\left[y_{k}^{3} e^{i \omega_{k} s} \int_{-\tau_{k_{n}}}^{-\tau_{k_{n}}+\delta} e^{2 i \omega_{k} \theta} d \theta+\bar{y}_{k}^{2} y_{k} e^{-3 i \omega_{k} s} \int_{-\tau_{k}}^{-\tau_{k_{n}}+\delta} e^{-2 i \omega_{k} \theta} d \theta+\right. \\
& \left.+2 y_{k}^{2} \bar{y}_{k} e^{-i \omega_{k} s}+y_{k}^{3} e^{i \omega_{k} s}+2 y_{k}^{2} \bar{y} k e^{-i \omega_{k} s}+y_{k} \bar{y}_{k}^{2} e^{-3 i \omega_{k} s}\right] \\
a_{21} & =\overline{a_{11}} \\
a_{22} & =\overline{a_{12}}
\end{aligned}
$$

Observando que $\int_{0}^{v_{k}} e^{\pi i \omega_{k} s} d s=0$ para todo $n \neq 0$, concluímos que

$$
\mathfrak{F}(0,0,0, W) \equiv 0
$$

Isto implica que

$$
\tilde{f}(s)=-\frac{k}{\delta} \int_{-\tau_{k_{n}}}^{-\tau_{k_{n}}+\delta}\left\{\left[1+9 U_{k}^{1}\right] \Phi_{1}(s) \Phi_{1}(s+\theta)+3 U_{k}^{4}\left[\Phi_{1}^{2}(s+\theta)+\Phi_{1}^{2}(s)\right]\right\} d \theta \in N(\mathbf{I})
$$

Para resolvermos a equação de bifurcação (4.6) numa vizinhança conveniente da origem, usaremos alguns lemas. 
Lema 4.1.

$$
\frac{\partial \mathfrak{F}(0,0,0, W)}{\partial(\mu, \gamma)}=v_{k}\left[\begin{array}{cc}
\operatorname{Re}\left(\dot{\lambda}\left(\tau_{k_{n}}\right)\right) & 0 \\
-\operatorname{Im}\left(\dot{\lambda}\left(\tau_{k_{n}}\right)\right) & -\omega_{k}
\end{array}\right]
$$

com $\dot{\lambda}\left(\tau_{k_{n}}\right)$ definido anteriormente.

Prova: Primeiramente vamos calcular $\frac{\partial \mathfrak{F}(0,0,0, W)}{\partial \mu}$.

Vemos que

$$
\begin{aligned}
N\left(0, \mu, 0, W_{t}\right) & =\frac{k}{\delta}\left\{\left(U_{k}+3 U_{k}^{5}\right) \int_{0}^{1} \int_{-\tau_{k_{n}}}^{-\tau_{k_{n}}+\delta} \dot{\Phi}_{1}(t+s) \mu d s d \theta\right. \\
& \left.-\left(1+9 U_{k}^{4}\right) \int_{-\tau_{k_{n}}}^{-\tau_{k_{n}}+\delta} \Phi_{1}(t) \Phi_{1}(t+s) d s-3 U_{k}^{4} \int_{-\tau_{k_{n}}}^{-\tau_{k_{n}}+\delta}\left[\Phi_{1}^{2}(t+s)+\Phi_{1}^{2}(t)\right] d s\right\}
\end{aligned}
$$

e portanto,

$$
\frac{\partial N\left(0, \mu, 0, W_{t}\right)}{\partial \mu}=\frac{k}{\delta}\left(U_{k}+3 U_{k}^{5}\right) \int_{-\tau_{k_{n}}}^{-\tau_{k_{n}}+\delta} \dot{\Phi}_{1}(t+s) d s
$$

Observamos que $\dot{\Phi}_{1}(t+\theta)=\frac{i \omega_{k}}{2}\left(\tilde{\Phi}_{1}(t+s)-\tilde{\Phi}_{2}(t+s)\right)$

Então,

$$
\begin{aligned}
& \frac{\partial \mathfrak{F}\left(0,0,0, W_{s}\right)}{\partial \mu}=\frac{k i \omega_{k}}{2 \delta} \int_{0}^{\nu_{k}} \int_{0}^{\pi} H^{-1}\left[\begin{array}{c}
\tilde{\Psi}_{1}(s) \\
\tilde{\Psi}_{2}(s)
\end{array}\right]\left(U_{k}+3 U_{k}^{5}\right) \int_{-\tau_{k_{n}}}^{-\tau_{k_{n}}+\delta}\left(\tilde{\Phi}_{1}(s+\theta)-\right. \\
& \left.\tilde{\Phi}_{2}(s+\theta)\right) d \theta d x d s
\end{aligned}
$$

Se chamarmos

$$
M=\left[\begin{array}{cc}
\tilde{\Psi}_{1}(s) \int_{-\tau_{k_{n}}+\delta}^{-\tau_{k_{n}}+\delta} \tilde{\Phi}_{1}(s+\theta) d \theta & -\tilde{\Psi}_{1}(s) \int_{-\tau_{n_{n}}}^{-\tau_{k_{n}}+\delta} \tilde{\Phi}_{2}(s+\theta) d \theta \\
\tilde{\Psi}_{2}(s) \int_{-\tau_{k_{n}}}^{-\tau_{k_{n}}+\delta} \tilde{\Phi}_{1}(s+\theta) d \theta & -\tilde{\Psi}_{2}(s) \int_{-\tau_{k_{n}}}^{-\tau_{k_{n}}+\delta} \tilde{\Phi}_{2}(s+\theta) d \theta
\end{array}\right]_{2 \times 2}
$$


então

$$
\frac{\partial \mathfrak{F}\left(0,0,0, W_{s}\right)}{\partial \mu}=\frac{k i \omega_{k}}{\delta} \int_{0}^{v_{k}} \int_{0}^{\pi}\left(U_{k}+3 U_{k}^{5}\right) H^{-1} M H\left(\begin{array}{l}
1 \\
0
\end{array}\right) d x d s
$$

É fácil ver que

$$
\frac{\partial \mathfrak{F}\left(0,0,0, W_{s}\right)}{\partial \mu}=H^{-1}\left[\begin{array}{cc}
v_{k} \dot{\lambda}\left(\tau_{k_{n}}\right) & 0 \\
0 & v_{k} \dot{\lambda}\left(\tau_{k_{n}}\right)
\end{array}\right] H\left(\begin{array}{l}
1 \\
0
\end{array}\right)
$$

e assim,

$$
\frac{\partial \mathfrak{F}\left(0,0,0, W_{s}\right)}{\partial \mu}=v_{k}\left[\begin{array}{c}
\operatorname{Re} \dot{\lambda}\left(\tau_{k_{n}}\right) \\
-\operatorname{Im} \dot{\lambda}\left(\tau_{k_{n}}\right)
\end{array}\right]
$$

Resta, agora, calcularmos $\frac{\partial \mathfrak{F}\left(0,0,0, W_{s}\right)}{\partial \gamma}$.

$$
\begin{aligned}
N\left(0,0, \gamma, W_{t}\right) & =\gamma A(k) \Phi_{1}(t)-\frac{\gamma k}{\delta}\left(U_{k}+3 U_{k}^{5}\right) \int_{-\tau_{k_{n}}}^{-\tau_{k_{n}}+\delta}\left(U_{k}+3 U_{k}^{5}\right)\left[\Phi_{1}(t+s)-\dot{\Phi}_{1}(t+s)\right] d s \\
& -\frac{k}{\delta}\left(1+9 U_{k}^{4}\right) \int_{-\tau_{k_{n}}}^{-\tau_{k_{n}}+\delta} \Phi_{1}(t) \Phi_{1}(t+s) d s-\frac{k}{\delta} 3 U_{k}^{4} \int_{-\tau_{k_{n}}}^{-\tau_{k_{n}}+\delta}\left[\Phi_{1}^{2}(t+s)+\Phi_{1}^{2}(t)\right] d s
\end{aligned}
$$

e portanto,

$$
\frac{\partial N\left(0,0, \gamma, W_{\iota}\right)}{\partial \gamma}=A(k) \Phi_{1}(t)-\frac{k}{\delta} \int_{-\tau_{k_{n}}}^{-\tau_{k_{n}}+\delta}\left(U_{k}+3 U_{k}^{5}\right)\left[\Phi_{1}(t+s)-\dot{\Phi}_{1}(t+s)\right] d s
$$

Daí,

$$
\begin{aligned}
& \frac{\partial \mathfrak{F}\left(0,0,0, W_{s}\right)}{\partial \gamma}=\int_{0}^{v_{k}} \int_{0}^{\pi} H^{-1}\left[\begin{array}{c}
\tilde{\Psi}_{1}(s) \\
\tilde{\Psi}_{2}(s)
\end{array}\right] A(k)\left[\begin{array}{ll}
\tilde{\Phi}_{1}(s) & \tilde{\Phi}_{2}(s)
\end{array}\right] H\left(\begin{array}{l}
1 \\
0
\end{array}\right) d x d s
\end{aligned}
$$

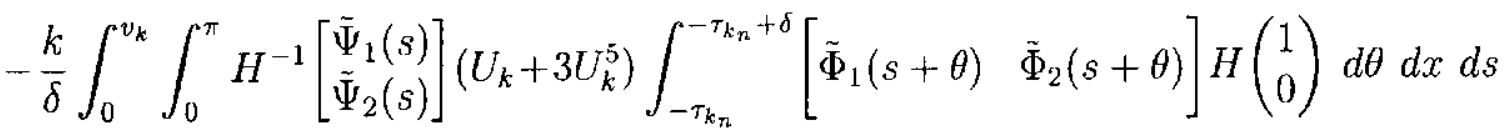

$$
\begin{aligned}
& -\frac{k}{\delta} \int_{0}^{v_{k}} \int_{0}^{\pi} H^{-1}\left[\begin{array}{c}
\tilde{\Psi}_{1}(s) \\
\tilde{\Psi}_{2}(s)
\end{array}\right]\left(U_{k}+3 U_{k}^{5}\right) \int_{-\tau_{k_{n}}}^{-\tau_{k_{n}}+\delta} \frac{i \omega_{k} \theta}{2}\left(\tilde{\Phi}_{1}(s+\theta)-\tilde{\Phi}_{2}(s+\theta)\right) d \theta d x d s
\end{aligned}
$$


Desenvolvendo as expressões e chamando

$$
M_{\theta}=\left[\begin{array}{ll}
\tilde{\Psi}_{1}(s) \int_{-\tau_{k_{n}}}^{-\tau_{k_{n}}+\delta} \tilde{\Phi}_{1}(s+\theta) \theta d \theta & -\tilde{\Psi}_{1}(s) \int_{-\tau_{k_{n}}}^{-\tau_{k_{n}}+\delta} \tilde{\Phi}_{2}(s+\theta) \theta d \theta \\
\tilde{\Psi}_{2}(s) \int_{-\tau_{k_{n}}}^{-\tau_{k_{n}}+\delta} \tilde{\Phi}_{1}(s+\theta) \theta d \theta & -\tilde{\Psi}_{2}(s) \int_{-\tau_{k_{n}}}^{-\tau_{k_{n}}+\delta} \tilde{\Phi}_{2}(s+\theta) \theta d \theta
\end{array}\right]_{2 \times 2}
$$

temos

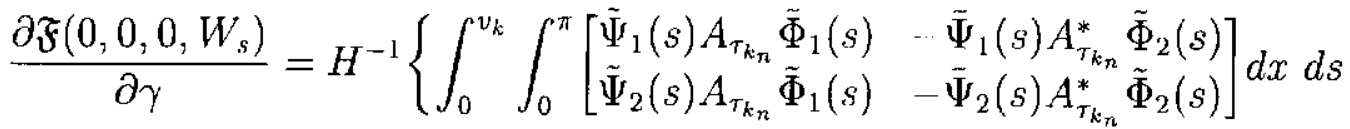

$$
\begin{aligned}
& \left.-\frac{k i \omega_{k}}{\delta} \int_{0}^{v_{k}} \int_{0}^{\pi}\left(U_{k}+3 U_{k}^{5}\right) M_{0} d x d s\right\} H\left(\begin{array}{l}
1 \\
0
\end{array}\right)
\end{aligned}
$$

que nos fornece

$$
\begin{aligned}
\frac{\partial \mathfrak{F}\left(0,0,0, W_{s}\right)}{\partial \gamma} & =H^{-1}\left[\begin{array}{cc}
\frac{i \omega_{k} v_{k}}{S_{k_{n}}} S_{k_{n}} & 0 \\
0 & \frac{-i \omega_{k} v_{k}}{\bar{S}_{k_{n}}} \bar{S}_{k_{n}}
\end{array}\right] H\left(\begin{array}{l}
1 \\
0
\end{array}\right) \\
& =-v_{k}\left[\begin{array}{c}
0 \\
\omega_{k}
\end{array}\right]
\end{aligned}
$$

o que conclui a prova do lema.

Lema 4.2. Seja

$$
f(\cdot)=-\frac{k}{\delta} \int_{-\tau_{k_{n}}}^{-\tau_{k_{n}}+\delta}\left\{\left[1+9 U_{k}^{4}\right] \Phi_{1}(\cdot) \Phi_{1}(\cdot+\theta)+3 U_{k}^{4}\left[\Phi_{1}^{2}(\cdot+\theta)+\Phi_{1}^{2}(\cdot)\right]\right\} d \theta
$$

Então, se $W_{k}=\mathcal{K}(f)$, 


$$
\begin{aligned}
W_{k}(t) & =\left(1+9 U_{k}^{4}\right)\left[\frac{1}{\delta} \int_{-\tau_{k_{n}}}^{-\tau_{k_{n}}+\delta} e^{i \omega_{k} \theta} d \theta Y_{k} e^{2 i \omega_{k} t}+\right. \\
& \left.\frac{1}{\delta} \int_{-\tau_{k_{n}}}^{-\tau_{k_{n}}+\delta} e^{-i \omega_{k} \theta} d \theta \bar{Y}_{k} e^{-2 i \omega_{k} t}+\frac{1}{\delta} \int_{\tau_{k_{n}}}^{-\tau_{k_{n}}+\delta} \cos \omega_{k} \theta d \theta Z_{k}\right]+ \\
& 3 U_{k}^{4}\left[\left(1+\frac{1}{\delta} \int_{-\tau_{k_{n}}}^{-\tau_{k_{n}}+\delta} e^{2 i \omega_{k} \theta} d \theta\right) Y_{k} e^{2 i \omega_{k} t}+\right. \\
& \left.\left(1+\frac{1}{\delta} \int_{-\tau_{k_{n}}}^{-\tau_{k_{n}}+\delta} e^{-2 i \omega_{k} \theta} d \theta\right) \bar{Y}_{k} e^{-2 i \omega_{k} t}+2 Z_{k}\right]+\Phi(t) b
\end{aligned}
$$

onde

$$
\begin{aligned}
& Y_{k}=\frac{k}{4}\left[A(k)-\frac{k}{\delta}\left(U_{k}+3 U_{k}^{5}\right) \int_{-\tau_{k_{n}}}^{-\tau_{k_{n}}+\delta} e^{2 i \omega_{k} \theta} d \theta-2 i \omega_{k}\right]^{-1} y_{k}^{2}, \\
& Z_{k}=\frac{k}{2}\left[A(k)-k\left(U_{k}+3 U_{k}^{5}\right)\right]^{-1} y_{k} \bar{y}_{k} e \\
& b=-\left(\Psi,\left(1+9 U_{k}^{4}\right)\left[\frac{1}{\delta} \int_{-\tau_{k_{n}}}^{-\tau_{k_{n}}+\delta} e^{i \omega_{k} \theta} d \theta Y_{k} e^{2 i \omega_{k} \cdot}+\right.\right. \\
& \left.+\frac{1}{\delta} \int_{-\tau_{k_{n}}}^{-\tau_{k_{n}}+\delta} e^{-i \omega_{k} \theta} d \theta \bar{Y}_{k} e^{-2 i \omega_{k} \cdot}+\frac{1}{\delta} \int_{-\tau_{k_{n}}}^{-\tau_{k_{n}}+\delta} \cos \omega_{k} \theta d \theta Z_{k}\right]+ \\
& +3 U_{k}^{4}\left[\left(1+\frac{1}{\delta} \int_{-\tau_{k_{n}}}^{-\tau_{k_{n}}+\delta} e^{2 i \omega_{k} \theta} d \theta\right) Y_{k} e^{2 i \omega_{k} \cdot}+\right. \\
& \left.\left.+\left(1+\frac{1}{\delta} \int_{\cdots \tau_{k_{n}}}^{-\tau_{k_{n}}+\delta} e^{-2 i \omega_{k} \theta} d \theta\right) \bar{Y}_{k} e^{-2 i \omega_{k} \cdot}+2 Z_{k}\right]\right)
\end{aligned}
$$

Prova: Obviamente $W_{k}(t)$ é $\omega_{k}$-periódica. Vamos verificar que $W_{k}(t)$ é solução de

$$
\dot{w}(t)=A(k) w(t)-\frac{k}{\delta} \int_{-\tau_{k_{n}}}^{-\tau_{k_{n}}+\delta}\left(U_{k}+3 U_{k}^{5}\right) w(t+s) d s+f(t)
$$

com $f(t)$ dada no enunciado do lema. De fato,

$$
\begin{aligned}
& A(k) w(t)-\frac{k}{\delta} \int_{-\tau_{k_{n}}}^{-\tau_{k_{n}}+\delta}\left(U_{k}+3 U_{k}^{5}\right) w(t+s) d s-\dot{w}(t)= \\
& =\left(1+9 U_{k}^{4}\right)\left[A(k)-\frac{k}{\delta} \int_{-\tau_{k_{n}}}^{-\tau_{k_{n}}+\delta}\left(U_{k}+3 U_{k}^{5}\right) e^{2 i \omega_{k} s} d s-2 i \omega_{k}\right] \frac{1}{\delta} \int_{-\tau_{k_{n}}}^{-\tau_{k_{n}}+\delta} e^{i \omega_{k} \theta} d \theta Y_{k} e^{2 i \omega_{k} t}
\end{aligned}
$$




$$
\begin{aligned}
& +\left(1+9 U_{k}^{4}\right)\left[A(k)-\frac{k}{\delta} \int_{-\tau_{k_{n}}}^{-\tau_{k_{n}}+\delta}\left(U_{k}+3 U_{k}^{5}\right) e^{-2 i \omega_{k} s} d s+2 i \omega_{k}\right] \frac{1}{\delta} \int_{-\tau_{k_{n}}}^{-\tau_{k_{n}}+\delta} e^{-i \omega_{k} \theta} d \theta \bar{Y}_{k} e^{-2 i \omega_{k} \ell} \\
& +\left(1+9 U_{k}^{4}\right)\left[A(k)-k\left(U_{k}+3 U_{k}^{5}\right)\right] \frac{1}{\delta} \int_{-\tau_{k_{n}}}^{-\tau_{k_{n}}+\delta} \cos \omega_{k} \theta d \theta Z_{k} \\
& +3 U_{k}^{4}\left[A(k)-\frac{k}{\delta} \int_{-\tau_{k_{n}}}^{-\tau_{k_{n}}+\delta}\left(U_{k}+3 U_{k}^{5}\right) e^{2 i \omega_{k} s} d s-2 i \omega_{k}\right]\left(1+\frac{1}{\delta} \int_{-\tau_{k_{n}}}^{-\tau_{k_{n}}+\delta} e^{2 i \omega_{k} \theta} d \theta\right) Y_{k} e^{2 i \omega_{k} t} \\
& +3 U_{k}^{4}\left[A(k)-\frac{k}{\delta} \int_{-\tau_{k_{n}}}^{-\tau_{k_{n}}+\delta}\left(U_{k}+3 U_{k}^{5}\right) e^{-2 i \omega_{k} s} d s+2 i \omega_{k}\right]\left(1+\frac{1}{\delta} \int_{-\tau_{k_{n}}}^{-\tau_{k_{n}}+\delta} e^{-2 i \omega_{k} \theta} d \theta\right) \bar{Y}_{k} e^{-2 i \omega_{k} t} \\
& +3 U_{k}^{4}\left[A(k)-k\left(U_{k}+3 U_{k}^{5}\right)\right] 2 Z_{k} \\
& +A(k) \Phi(t) b-\frac{k}{\delta} \int_{-\tau_{k_{n}}}^{-\tau_{k_{n}}+\delta}\left(U_{k}+3 U_{k}^{5}\right) \Phi(t+s) b d s-\dot{\Phi}(t) b= \\
& =\left(1+9 U_{k}^{1}\right) \frac{k}{4} y_{k}^{2} \frac{1}{\delta} \int_{-\tau_{k_{n}}}^{-\tau_{k_{n}}+\delta} e^{i \omega_{k} \theta} d \theta e^{2 i \omega_{k} t}+\left(1+9 U_{k}^{4}\right) \frac{k}{4} \bar{y}_{k}^{2} \frac{1}{\delta} \int_{-\tau_{k_{n}}}^{-\tau_{k_{n}}+\delta} e^{-i \omega_{k} \theta} d \theta e^{-2 i \omega_{k} t} \\
& +\left(1+9 U_{k}^{4}\right) \frac{k}{2} y_{k} \bar{y}_{k} \frac{1}{\delta} \int_{-\tau_{k_{n}}}^{-\tau_{k_{n}}+\delta} \cos \omega_{k} \theta d \theta+3 U_{k}^{4} \frac{k}{4} y_{k}^{2}\left(1+\frac{1}{\delta} \int_{-\tau_{k_{n}}}^{-\tau_{k_{n}}+\delta} e^{2 i \omega_{k} \theta} d \theta\right) e^{2 i \omega_{k} t} \\
& \left.+\frac{1}{\delta} \int_{-\tau_{k_{n}}}^{-\tau_{k_{n}}+\delta} e^{-2 i \omega_{k} \theta} d \theta\right) e^{-2 i \omega_{k} t}+3 U_{k}^{4} k y_{k} \bar{y}_{k}=-f(t)
\end{aligned}
$$

observando que $\Phi(t) b$ é solução da equação homogênea.

Também, lembrando que $(\Psi, \Phi)=I$, e a definição de b, temos que $\left(\Psi, W_{k}\right)=0$. Portanto, $W_{k}=\mathcal{K}(f)$.

Gostaríamos, também, de provar que a bifurcação de Hopf saindo de $U_{k}$ ocorre quando o retardo $\tau$ passa monotonicamente crescente através de cada $\tau_{k_{n}}$. Para isso necessitamos dos seguintes lemas.

Lema 4.3. Sejam $Y_{k}$ e $Z_{k}$ como definidos no lema (4.2). Então,

$$
\left.\frac{\partial \mathfrak{F}(c, 0,0, W)}{\partial c}\right|_{c=0}=\left[\begin{array}{c}
T(k) \\
T^{*}(k)
\end{array}\right]
$$

onde $T(k)=T_{1}(k)+T_{2}(k)+T_{3}(k)+T_{4}(k)+T_{5}(k)+T_{6}(k), e$ 


$$
\begin{aligned}
& T_{1}(k)=-\frac{9}{4} k v_{k} \int_{0}^{\pi} U_{k}^{3} R e\left[\frac{1}{S_{k_{n}}} y_{k}^{3} \bar{y}_{k} \frac{1}{\delta} \int_{-\tau_{k_{n}}}^{-\tau_{k_{n}}+\delta}\left(e^{-i \omega_{k} \theta}+2 e^{i \omega_{k} \theta}+e^{2 i \omega_{k} \theta}+2\right) d \theta\right] d x \\
& T_{2}(k)=-\frac{3}{4} k v_{k} \int_{0}^{\pi} U_{k}^{3} \operatorname{Re}\left[\frac{1}{S_{k_{n}}} y_{k}^{3} \bar{y}_{k} \frac{1}{\delta} \int_{-\tau_{k_{n}}}^{-\tau_{k_{n}}+\delta}\left(e^{i \omega_{k} \theta}+1\right) d \theta\right] d x \\
& T_{3}(k)=-k v_{k} \int_{0}^{\pi}\left(1+9 U_{k}^{4}\right)^{2} \operatorname{Re}\left[\frac { 1 } { S _ { k _ { n } } } \left\{Z_{k} y_{k}^{2} \frac{1}{\delta} \int_{-\tau_{k_{n}}}^{-\tau_{k_{n}}+\delta}\left(e^{i \omega_{k} \theta}+1\right) d \theta \frac{1}{\delta} \int_{-\tau_{k_{n}}}^{-\tau_{k_{n}}+\delta} \cos \omega_{k} \theta d \theta\right.\right. \\
& \left.\left.+Y_{k} y_{k} \bar{y}_{k} \frac{1}{\delta} \int_{-\tau_{k_{n}}}^{-\tau_{k_{n}}+\delta}\left(e^{-i \omega_{k} \theta}+e^{2 i \omega_{k} \theta}\right) d \theta \frac{1}{\delta} \int_{-\tau_{k_{n}}}^{-\tau_{k_{n}}+\delta} e^{i \omega_{k} \theta} d \theta\right\}\right] d x \\
& T_{4}(k)=-3 k v_{k} \int_{0}^{\pi}\left(1+9 U_{k}^{4}\right) U_{k}^{4} \operatorname{Re}\left[\frac { 1 } { S _ { k _ { n } } } \left\{2 Z_{k} y_{k}^{2} \frac{1}{\delta} \int_{-\tau_{k_{n}}}^{-\tau_{k_{n}}+\delta}\left(e^{i \omega_{k} \theta}+1\right) d \theta\right.\right. \\
& \left.\left.+Y_{k} y_{k} \bar{y}_{k}\left(1+\frac{1}{\delta} \int_{-\tau_{k_{n}}}^{-\tau_{k_{n}}+\delta} e^{2 i \omega_{k} \theta} d \theta\right)\left(\frac{1}{\delta} \int_{-\tau_{k_{n}}}^{\cdots \tau_{k_{n}}+\delta}\left(e^{-i \omega_{k} \theta}+e^{2 i \omega_{k} \theta}\right) d \theta\right)\right\}\right] d x
\end{aligned}
$$$$
T_{5}(k)=-6 v_{k} \int_{0}^{\pi}\left(1+9 U_{k}^{4}\right) U_{k}^{4} \operatorname{Re}\left[\frac { 1 } { S _ { k _ { n } } } \left\{Z_{k} y_{k}^{2} \frac{1}{\delta} \int_{\tau_{k_{n}}}^{-\tau_{k_{n}}+\delta}\left(e^{i \omega_{k} \theta}+1\right) d \theta\right.\right.
$$$$
\frac{1}{\delta} \int_{-\tau_{k_{n}}}^{-\tau_{k_{n}}+\delta} \cos \omega_{k} \theta d \theta+Y_{k} y_{k} \bar{y}_{k}\left(1+\frac{1}{\delta} \int_{-\tau_{k_{n}}}^{-\tau_{k_{n}}+\delta}\left(e^{i \omega_{k} \theta}+1\right) d \theta\right)
$$$$
\left.\left.\left(\frac{1}{\delta} \int_{-\tau_{k_{n}}}^{-\tau_{k_{n}}+\delta} e^{i \omega_{k} \theta} d \theta\right)\right\}\right] d x
$$

$$
\begin{aligned}
& T_{6}(k)=-18 k v_{k} \int_{0}^{\pi} U_{k}^{8} R e\left[\frac { 1 } { S _ { k _ { n } } } \left\{2 Z_{k} y_{k}^{2} \frac{1}{\delta} \int_{\tau_{k_{n}}}^{-\tau_{k_{n}}+\delta}\left(e^{i \omega_{k} \theta}+1\right) d \theta\right.\right. \\
& \left.\left.+Y_{k} y_{k} \bar{y}_{k}\left(1+\frac{1}{\delta} \int_{-\tau_{k_{n}}}^{-\tau_{k_{n}}+\delta}\left(e^{i \omega_{k} \theta}+1\right) d \theta\right)\left(1+\frac{1}{\delta} \int_{-\tau_{k_{n}}}^{-\tau_{k_{n}}+\delta} e^{2 i \omega_{k} \theta} d \theta\right)\right\}\right] d x
\end{aligned}
$$

$e T^{*}(k)=T(k)$, trocando Re por -Im em todas as expressões. 
Prova: Vemos que

$$
\begin{aligned}
& N\left(c, 0,0, W_{k}\right)=-\frac{k}{\delta} \int_{-\tau_{k_{n}}}^{-\tau_{k_{n}}+\delta}\left[\Phi_{1}(t)+c W_{k}(t)\right]\left[\Phi_{1}(t+s)+c W_{k}(t+s)\right] d s \\
& -\frac{k}{\delta} \int_{-\tau_{k_{n}}}^{-\tau_{k_{n}}+\delta} \sum_{p, p^{\prime}}^{2}\left(\begin{array}{l}
3 \\
p
\end{array}\right)\left(\begin{array}{c}
3 \\
p^{\prime}
\end{array}\right) U_{k}^{p \cdot+p^{\prime}} c^{4-p-p^{\prime}}\left[\Phi_{1}(t)+c W_{k}(t)\right]^{3-p}\left[\Phi_{1}(t+s)+c W_{k}(t+s)\right]^{3-p^{\prime}} d s \\
& -\frac{k}{\delta} \int_{-\tau_{k_{n}}}^{-\tau_{k_{n}}+\delta} U_{k}^{3} c\left\{\left[\Phi_{1}(t+s)+c W_{k}(t+s)\right]^{3}+\left[\Phi_{1}(t)+c W_{k}(t)\right]^{3}\right\} d s \\
& -\frac{k}{\delta} \int_{-\tau_{k_{n}}}^{-\tau_{k_{n}}+\delta} 3 U_{k}^{4}\left\{\left[\Phi_{1}(t+s)+c W_{k}(t+s)\right]^{2}+\left[\Phi_{1}(t)+c W_{k}(t)\right]^{2}\right\} d s
\end{aligned}
$$

e portanto

$$
\begin{aligned}
& \left.\frac{\partial N\left(c, 0,0, W_{k}\right)}{\partial c}\right|_{c=0}=-\frac{k}{\delta}\left(1+9 U_{k}^{4}\right) \int_{-\tau_{k_{n}}}^{-\tau_{k_{n}}+\delta}\left[\Phi_{1}(t+s) W_{k}(t)+\Phi_{1}(t) W_{k}(t+s)\right] d s \\
& -\frac{k}{\delta} 9 U_{k}^{3} \int_{-\tau_{k_{n}}}^{-\tau_{k_{n}}+\delta}\left[\Phi_{1}^{2}(t) \Phi(t+s)+\Phi_{1}(t) \Phi_{1}^{2}(t+s)\right] d s-\frac{k}{\delta} U_{k}^{3} \int_{-\tau_{k_{n}}}^{-\tau_{k_{n}}+\delta}\left[\Phi_{1}^{3}(t+s)\right. \\
& \left.+\Phi_{1}^{3}(t)\right] d s-\frac{k}{\delta} 6 U_{k}^{4} \int_{-\tau_{k_{n}}}^{-\tau_{k_{n}}+\delta}\left[\Phi_{1}(l+s) W_{k}(t+s)+\Phi_{1}(t) W_{k}(t)\right] d s
\end{aligned}
$$

\section{Então,}

$$
\begin{aligned}
& \left.\frac{\partial \mathfrak{F}(c, 0,0, W)}{\partial c}\right|_{c=0}=\int_{0}^{v_{k}}\left\langle\Psi(s),-\frac{k}{\delta}\left(1+9 U_{k}^{4}\right) \int_{-\tau_{k_{n}}}^{-\tau_{k_{n}}+\delta}\left[\Phi_{1}(s+\theta) W_{k}(s)\right.\right. \\
& \left.\left.+\Phi_{1}(s) W_{k}(s+\theta)\right] d \theta\right\rangle^{*} d s \\
& +\int_{0}^{v_{k}}\left\langle\Psi(s),-\frac{k}{\delta} 9 U_{k}^{3} \int_{\tau_{k_{n}}}^{-\tau_{k_{n}}+\delta}\left[\Phi_{1}^{2}(s) \Phi(s+\theta)+\Phi_{1}(s) \Phi_{1}^{2}(s+\theta)\right] d \theta\right\rangle^{*} d s \\
& +\int_{0}^{v_{k}}\left\langle\Psi(s),-\frac{k}{\delta} U_{k}^{3} \int_{-\tau_{k_{n}}}^{-\tau_{k_{n}}+\delta}\left[\Phi_{1}^{3}(s+\theta)+\Phi_{1}^{3}(s)\right] d \theta\right\rangle^{*} d s \\
& +\int_{0}^{v_{k}}\left\langle\Psi(s),-\frac{k}{\delta} 6 U_{k}^{4} \int_{-\tau_{k_{n}}}^{-\tau_{k_{n}}+\delta}\left[\Phi_{1}(s+\theta) W_{k}(s+\theta)+\Phi_{1}(s) W_{k}(s)\right] d \theta\right\rangle^{*} d s
\end{aligned}
$$

Vamos colocar 


$$
W_{k}(s)=\left(1+9 U_{k}^{4}\right) W_{k}^{1}(s)+3 U_{k}^{4} W_{k}^{2}(s)+\Phi(s) b
$$

onde

$$
\begin{aligned}
& W_{k}^{1}(s)=\frac{1}{\delta} \int_{-\tau_{k_{n}}}^{-\tau_{k_{n}}+\delta} e^{i \omega_{k} \theta} d \theta Y_{k} e^{2 i \omega_{k} t}+\frac{1}{\delta} \int_{-\tau_{k_{n}}}^{-\tau_{k_{n}}+\delta} e^{-i \omega_{k} \theta} d \theta \bar{Y}_{k} e^{-2 i \omega_{k} t} \\
& +\frac{1}{\delta} \int_{-\tau_{k_{n}}}^{-\tau_{k_{n}}+\delta} \cos \omega_{k} \theta d \theta Z_{k} \\
& W_{k}^{2}=\left(1+\frac{1}{\delta} \int_{-\tau_{k_{n}}}^{-\tau_{k_{n}}+\delta} e^{2 i \omega_{k} \theta} d \theta\right) Y_{k} e^{2 i \omega_{k} t}+\left(1+\frac{1}{\delta} \int_{-\tau_{k_{n}}}^{-\tau_{k_{n}}+\delta} e^{-2 i \omega_{k} \theta} d \theta\right) \bar{Y}_{k} e^{-2 i \omega_{k} t}+2 Z_{k}
\end{aligned}
$$

Abrindo cada uma das parcelas, temos que:

$$
\left.\frac{\partial \mathfrak{F}(c, 0,0, W)}{\partial c}\right|_{c=0}=T_{1}+T_{2}+T_{3}+T_{4}+T_{5}+T_{6}+T_{7}+T_{8}
$$

onde

$$
\begin{gathered}
T_{1}=\int_{0}^{v_{k}}\left\langle\Psi(s),-\frac{k}{\delta} 9 U_{k}^{3} \int_{-\tau_{k_{n}}}^{-\tau_{k_{n}}+\delta}\left[\Phi_{1}^{2}(s) \Phi(s+\theta)+\Phi_{1}(s) \Phi_{1}^{2}(s+\theta)\right] d \theta\right\rangle^{*} d s \\
T_{2}=\int_{0}^{v_{k}}\left\langle\Psi(s),-\frac{k}{\delta} U_{k}^{3} \int_{-\tau_{k_{n}}}^{-\tau_{k_{n}}+\delta}\left[\Phi_{1}^{3}(s+\theta)+\Phi_{1}^{3}(s)\right] d \theta\right\rangle^{*} d s \\
T_{3}=\int_{0}^{v_{k}}\left\langle\Psi(s),-\frac{k}{\delta}\left(1+9 U_{k}^{4}\right)^{2} \int_{-\tau_{k_{n}}}^{-\tau_{k_{n}}+\delta}\left[\Phi_{1}(s+\theta) W_{k}^{1}(s)\right.\right. \\
\left.\left.+\Phi_{1}(s) W_{k}^{1}(s+\theta)\right] d \theta\right\rangle^{*} d s
\end{gathered}
$$




$$
\begin{gathered}
T_{4}=\int_{0}^{v_{k}}\left\langle\Psi(s),-\frac{k}{\delta}\left(1+9 U_{k}^{4}\right) 3 U_{k}^{4} \int_{-\tau_{k_{n}}}^{-\tau_{k_{n}}+\delta}\left[\Phi_{1}(s+\theta) W_{k}^{2}(s)\right.\right. \\
\left.\left.+\Phi_{1}(s) W_{k}^{2}(s+\theta)\right] d \theta\right\rangle^{*} d s \\
T_{5}=\int_{0}^{v_{k}}\left\langle\Psi(s),-\frac{k}{\delta} 6 U_{k}^{4}\left(1+9 U_{k}^{4}\right) \int_{-\tau_{k_{n}}}^{-\tau_{k_{n}}+\delta}\left[\Phi_{1}(s+\theta) W_{k}^{1}(s+\theta)\right.\right. \\
T_{6}=\int_{0}^{v_{k}}\left\langle\Psi(s),-\frac{k}{\delta} 18 U_{k}^{4} \int_{-\tau_{k_{n}}}^{-\tau_{k_{n}}+\delta}\left[\Phi_{1}(s+\theta) W_{k}^{2}(s+\theta)+\Phi_{k}^{1}(s)\right] d \theta\right\rangle^{*} d s \\
T_{7}=\int_{0}^{v_{k}}\left\langle\Psi(s),-\frac{k}{\delta}\left(1+9 U_{k}^{4}\right) \int_{-\tau_{k_{n}}}^{-\tau_{k_{n}}+\delta}\left[\Phi_{1}^{2}(s)\right] d \theta\right\rangle^{*} d s \\
T_{8}=\int_{0}^{v_{k}}\left\langle\Psi(s),-\frac{k}{\delta} 6 U_{k}^{4} \int_{-\tau_{k_{n}}}^{\sim \tau_{k_{n}}+\delta}\left[\Phi_{1}(s+\theta) \Phi(s+\theta) b+\Phi_{1}(s) \Phi(s) b\right] d \theta\right\rangle^{*} d s \\
\left.\left.+\Phi_{1}(s) \Phi(s+\theta) b\right] d \theta\right\rangle^{*} d s
\end{gathered}
$$

Desenvolvendo estas expressões vemos que $T_{1}, \ldots, T_{6}$ são as expressões que desejamos e $T_{7}=T_{8}=0$.

Lema 4.4. Para $0<k-1 \ll 1, T(k)<0$.

Prova: Para estimarmos $T(k)$, primeiramente precisamos calcular $\lim _{k \rightarrow 1^{+}} Y_{k}(k-1)$ $e \lim _{k \rightarrow 1^{+}} Z_{k}(k-1)$.

Vamos calcular, então, $\lim _{k \rightarrow 1^{+}} Y_{k}(k-1)$

Decompomos $Y_{k}$ daseguinte forma:

$$
Y_{k}=\frac{1}{k-1}\left[m_{k} \operatorname{sen}(\cdot)+(k-1) Y_{k}^{*}\right]
$$


$\operatorname{com} m_{k} \in \mathbb{C} e \int_{0}^{\pi} Y_{k}^{*}(x) \operatorname{sen}(x) d x=0$

Observamos que $A(k)$ pode ser escrita da seguinte forma $A(k)=D^{2}+1+(k-$ 1) $-k\left(U_{k}+3 U_{k}^{5}\right)$ e temos:

$\left(D^{2}+1\right) \operatorname{sen}(\cdot)=0, \omega_{k}=(k-1) \rho_{k}$ e $U_{k}=\alpha_{k}(k-1)[\operatorname{sen}(\cdot)+O(k-1)]$

Queremos estimar $m_{k}$ e $Y_{k}^{*}$ quando $k \rightarrow 1+$.

$$
\begin{array}{r}
\frac{1}{(k-1)}\left[m_{k} \operatorname{sen}(\cdot)+(k-1) Y_{k}^{*}\right]=\frac{k}{4}\left[A(k)-\frac{k}{\delta}\left(U_{k}+3 U_{k}^{5}\right) \int_{-\tau_{k_{n}}}^{-\tau_{k_{n}}+\delta} e^{2 i \omega_{k} \theta} d \theta\right. \\
\left.-2 i \omega_{k}\right]^{-1} y_{k}^{2}
\end{array}
$$

$$
\frac{1}{(k-1)}\left[A(k)-\frac{k}{\delta}\left(U_{k}+3 U_{k}^{5}\right) \int_{-\tau_{k_{n}}}^{-\tau_{k_{n}}+\delta} e^{2 i \omega_{k} \theta} d \theta-2 i \omega_{k}\right]\left[m_{k} \operatorname{sen}(\cdot)+(k-1) Y_{k}^{*}\right]=\frac{k}{4} y_{k}^{2}
$$

Substituindo $A(k), \omega_{k}, U_{k}$ e colocando $(k-1)$ em evidência, vem:

$$
\left(D^{2}+1\right) Y_{k}^{*}+m_{k} J_{k} \operatorname{sen}(\cdot)+(k-1) J_{k} Y_{k}^{*}=\frac{k}{4} y_{k}^{2}
$$

onde $J_{k}(x)=1-2 i \rho_{k}-k\left(1+\frac{1}{\delta} \int_{-\tau_{k_{n}}}^{-\tau_{k_{n}}+\delta} e^{2 i \omega_{k} \theta} d \theta\right) \alpha_{k}(\operatorname{sen}(\cdot)+O(k-1))$

Multiplicando por sen $(\cdot)$ e integrando de 0 a $\pi$,vem:

$$
m_{k}=\frac{\frac{k}{4} \int_{0}^{\pi} y_{k}^{2}(x) \operatorname{sen}(x) d x-(k-1) \int_{0}^{\pi} J_{k}(x) Y_{k}^{*}(x) \operatorname{sen}(x) d x}{\int_{0}^{\pi} J_{k}(x) \operatorname{sen}^{2}(x) d x}
$$

É fácil ver que $\lim _{k \rightarrow 1^{+}} \int_{0}^{\pi} J_{k}(x) \operatorname{sen}^{2}(x) d x=(1-2 i) \int_{0}^{\pi} \operatorname{sen}^{2}(x) d x$ e que $Y_{k}^{*} e^{\prime}$ limitado quando $k \rightarrow 1^{+}$. Então: 


$$
m_{k}=\frac{k \int_{0}^{\pi} \operatorname{sen}^{3}(x) d x}{(1-2 i) \int_{0}^{\pi} \operatorname{sen}^{2}(x) d x}=\frac{(1+2 i) k \int_{0}^{\pi} \operatorname{sen}^{3}(x) d x}{20 \int_{0}^{\pi} \operatorname{sen}^{2}(x) d x}
$$

E portanto,

$$
\lim _{k \rightarrow 1^{+}} Y_{k}(k-1)=\frac{(1+2 i) k \int_{0}^{\pi} \operatorname{sen}^{3}(x) d x}{20 \int_{0}^{\pi} \operatorname{sen}^{2}(x) d x} \operatorname{sen}(\cdot)
$$

Com o mesmo raciocínio, mostramos que

$$
\lim _{k \rightarrow 1^{+}} Z_{k}(k-1)=\frac{-\int_{0}^{\pi} \operatorname{sen}^{3}(x) d x}{2 \int_{0}^{\pi} \operatorname{sen}^{2}(x) d x} \operatorname{sen}(\cdot)
$$

Näo é difícil ver que $T_{1}, T_{2}, T_{4}, T_{5}$ e $T_{6}$ convergem para 0 quando $k \rightarrow 1^{+}$. Para $T_{3}$ temos:

$$
\begin{aligned}
& T_{3}(k)=-k v_{k} \int_{0}^{\pi}\left(1+9 U_{k}^{4}\right)^{2} \operatorname{Re}\left[\frac { 1 } { S _ { k _ { n } } } \left\{Z_{k} y_{k}^{2} \frac{1}{\delta} \int_{-\tau_{k_{n}}}^{-\tau_{k_{n}}+\delta}\left(e^{i \omega_{k} \theta}+1\right) d \theta \frac{1}{\delta} \int_{-\tau_{k_{n}}}^{-\tau_{k_{n}}+\delta} \cos \omega_{k} \theta d \theta\right.\right. \\
& \left.\left.+Y_{k} y_{k} \bar{y}_{k} \frac{1}{\delta} \int_{-\tau_{k_{n}}}^{-\tau_{k_{n}}+\delta}\left(e^{-i \omega_{k} \theta}+e^{2 i \omega_{k} \theta}\right) d \theta \frac{1}{\delta} \int_{-\tau_{k_{n}}}^{-\tau_{k_{n}}+\delta} e^{i \omega_{k} \theta} d \theta\right\}\right] d x \\
& =\frac{-k v_{k}}{(k-1)} \int_{0}^{\pi}\left(1+9 U_{k}^{4}\right)^{2} \operatorname{Re}\left[\frac { 1 } { S _ { k _ { n } } } \left\{Z_{k}(k-1) y_{k}^{2} \frac{1}{\delta} \int_{-\tau_{k_{n}}}^{-\tau_{k_{n}}+\delta}\left(e^{i \omega_{k} \theta}+1\right) d \theta \frac{1}{\delta} \int_{-\tau_{k_{n}}}^{-\tau_{k_{n}}+\delta} \cos \omega_{k} \theta d \theta\right.\right. \\
& \left.\left.+Y_{k}(k-1) y_{k} \bar{y}_{k} \frac{1}{\delta} \int_{-\tau_{k_{n}}}^{-\tau_{k_{n}}+\delta}\left(e^{-i \omega_{k} \theta}+e^{2 i \omega_{k} \theta}\right) d \theta \frac{1}{\delta} \int_{-\tau_{k_{n}}}^{-\tau_{k_{n}}+\delta} e^{i \omega_{k} \theta} d \theta\right\}\right] d x
\end{aligned}
$$

Pelas estimativas acima e observando que $\lim _{k \rightarrow 1^{+}} \int_{-\tau_{k_{n}}}^{-\tau_{k_{n}}+\delta} \cos \omega_{k} \theta d \theta=0$, temos que

$$
\lim _{k \rightarrow 1^{+}} Z_{k}(k-1) y_{k}^{2} \frac{1}{\delta} \int_{-\tau_{k_{n}}}^{-\tau_{k_{n}}+\delta}\left(e^{i \omega_{k} \theta}+1\right) d \theta \frac{1}{\delta} \int_{-\tau_{k_{n}}}^{-\tau_{k_{n}}+\delta} \cos \omega_{k} \theta d \theta=0
$$

e assim, para $k \approx 1$, temos: 


$$
\begin{aligned}
T_{3} & \approx \frac{-v_{k}}{k-1} \int_{0}^{\pi} \operatorname{Re}\left[\frac{S_{k_{n}}}{\left|S_{k_{n}}\right|^{2}} \frac{(1+2 i) \int_{0}^{\pi} \operatorname{sen}^{3}(x) d x}{20 \int_{0}^{\pi} \operatorname{sen}^{2}(x) d x} \operatorname{sen}^{3}(x)(1+i)\right] d x \\
& =\frac{-v_{k}}{(k-1)} \frac{1}{\left|S_{k_{n}}\right|^{2}}\left(\frac{3\left(2 n \pi+\frac{\pi}{2}\right)}{20}-1\right)\left(\int_{0}^{\pi} \operatorname{sen}^{3}(x) d x\right)^{2}<0
\end{aligned}
$$

Enunciamos, agora, o teorema principal:

Teorema 4.5. Para cada $k$ fixo, $k \in\left(1, k^{*}\right)$ uma bifurcação de Hopf ocorre quando o retardo $\tau$ passa crescentemente por cada ponto $\tau_{k_{n}}, n=0,1,2, \ldots$ Especificamente, para cada $\tau_{k_{n}}$, existe um $\delta_{k_{n}}>0$ tal que para cada $\tau \in\left(\tau_{k_{n}}, \tau_{k_{n}}+\delta_{k_{n}}\right)$ a equação (1) tern uma solução periódica $U_{k_{n}, r}$ próxima de $U_{k}$ com período $T\left(\tau_{k_{n}}\right) \approx \frac{2 \pi}{\omega_{k}}$.

Prova: Sabemos por (4.8) que $\mathfrak{F}(0,0,0, W) \equiv 0$, e por (4.1)

$$
\frac{\partial \mathfrak{F}(0,0,0, W)}{\partial(\mu, \gamma)}=v_{k}\left[\begin{array}{cc}
\operatorname{Re}\left(\dot{\lambda}\left(\tau_{k_{n}}\right)\right) & 0 \\
-\operatorname{Im}\left(\dot{\lambda}\left(\tau_{k_{n}}\right)\right) & -\omega_{k}
\end{array}\right]
$$

Como Re $\dot{\lambda}\left(\tau_{k_{n}}\right)>0$, existe uma vizinhança $B \subset \mathbb{R}$ da origem, uma vizinhança $V_{0} \subseteq \mathcal{P}_{v_{k}}^{1}$ de $W_{k}, c_{0}>0$, funções continuamente diferenciáveis $\mu:\left[-c_{0}, c_{0}\right] \times$ $V_{0} \rightarrow B, \gamma:\left[-c_{0}, c_{0}\right] \times V_{0} \rightarrow B$ tais que $\mu\left(0, W_{k}\right)=\gamma\left(0, W_{k}\right)=0$ e para cada $(c, W) \in\left[-c_{0}, c_{0}\right] \times V_{0},(\mu, \gamma) \in B \times B$

$$
\mathfrak{F}(c, \mu, \gamma, W)=0
$$

se e somente se

$$
\mu=\mu(c, W) \gamma=\gamma(c, W)
$$

Definimos,

$$
\Omega(c, W)=W-\mathcal{K}(N(c, \mu(c, W), \gamma(c, W), W))
$$

Entäo $\Omega\left(0, W_{k}\right)=0$. Por (4.8),

$$
\frac{\partial \mathfrak{F}\left(0,0,0, W_{k}\right)}{\partial W}=0
$$


Derivamos a equação (4.10) em relação a $W$ e calculamos em $c=0$, obtendo $\left.\frac{\partial \Omega}{\partial W}(c, W)\right|_{c=0}=I$

Assim, $\frac{\partial \Omega}{\partial W}\left(0, W_{k}\right): \mathcal{P}_{v_{k}}^{1} \rightarrow \mathcal{P}_{v_{k}}^{1}$ é bijetora. Logo, existe $c_{1} \in\left(0, c_{0}\right], V_{1} \subseteq V_{0}$ vizinhança de $W_{k}$ e $W^{*}:\left[-c_{1}, c_{1}\right] \rightarrow V_{1}$ tal que $W^{*}(0)=W_{k}$ e para $(c, W) \in$ $\left[-c_{1}, c_{1}\right] \times V_{1}, \Omega(c, W)=0$ se, e somente se, $W=W^{*}(c)$.

Portanlo, existe soluçẫo v veriódica para a equação (4.2), com $W(t)$ próxima ao zero para $\alpha, \beta$ suficientemente pequenos se, e somente se,

$$
W(t)=c \Phi_{1}(t)+[\mathcal{K} G(\alpha, \beta, w)](t)
$$

$\operatorname{com} \alpha=c \mu\left(c, W^{*}(c)\right), \beta=c \gamma\left(c, W^{*}(c)\right)$, para algum $c \in\left[-c_{1}, c_{1}\right]$, com $W^{*}(c, t)=$ $W^{*}(c)(t)$

$$
\operatorname{Sejam} \mu^{*}(c)=\mu\left(c, W^{*}(c)\right), \gamma^{*}(c)=\gamma\left(c, W^{*}(c)\right) .
$$

Como $\mathfrak{F}\left(c, \mu^{*}(c), \gamma^{*}(c), W^{*}(c)\right) \equiv 0$, para $c \in\left[-c_{1}, c_{1}\right]$, diferenciando ambos os lados da equação acima em $c$ e calculando em $c=0$, temos:

$$
\frac{\partial \mathfrak{F}\left(0,0,0, W_{k}\right)}{\partial c}+\frac{\partial \mathfrak{F}\left(0,0,0, W_{k}\right)}{\partial(\mu, \gamma)}\left[\begin{array}{l}
\frac{d}{d c} \mu^{*}(0) \\
\frac{d}{d c} \gamma^{*}(0)
\end{array}\right]=0
$$

ou seja,

$$
\begin{gathered}
{\left[\begin{array}{c}
\frac{d \mu^{*}}{d c}(0) \\
\frac{d \gamma^{*}}{d c}(0)
\end{array}\right]=-\frac{\partial \mathfrak{F}\left(0,0,0, W_{k}\right)^{-1}}{\partial(\mu, \gamma)} \frac{\partial \mathfrak{F}\left(0,0,0, W_{k}\right)}{\partial c}} \\
=\left[\begin{array}{c}
\frac{-T(k)}{\operatorname{Re} \dot{\lambda}\left(\tau_{k_{n}}\right)} \\
I m \dot{\lambda}\left(\tau_{k_{n}}\right) T(k)+\operatorname{Re} \dot{\lambda}\left(\tau_{k_{n}}\right) T^{*}(k)
\end{array}\right] \\
\text { Logo, } \frac{d \mu^{*}}{d c}(0)=\frac{-T(k)}{\operatorname{Re} \dot{\lambda}\left(\tau_{k_{n}}\right)}>0
\end{gathered}
$$


Desta forma, $\alpha=c \mu^{*}=\frac{-T(k)}{\operatorname{Re} \dot{\lambda}\left(\tau_{k_{n}}\right)} c^{2}+O\left(c^{3}\right)>0$

Assim, $\tau-\tau_{k_{n}}=\alpha>0$, e provamos que a bifurcação de Hopf ocorre quando o retardo $\tau$ passa crescentemente através de cada $\tau_{k_{n}}$. 


\section{Bibliografia}

[1] Santos, J.S. \& Bená, M.A., Hopf Bifurcation For a Delay Reaction-Diffusion Equation With Negative Feedback, to appear.

[2] Busenberg, S. \& Huang, W., Stability and Hopf Bifurcation for a Population Delay Model with Diffusion Effects, Journal of Differential Equations, 124, 80-107, [1996].

[3] Nussbaum, R.D., Periodic Solutions of Nonlinear Autonomous Functional Differential Equations, Lecture Notes in Math., 730, Springer, Berlin, [1979].

[4] Hale, J.K. \& Lunel, S.M.V, Introduction to Functional Differential Equations, Springer-Verlag, New York, [1993].

[5] Green,D \& Stech,H.W., Diffusion and hereditary effects in a class of population models, Differential Equations and Applications in Ecology Epidemics and Populations Problems, 19-28, Academics Press, [1981].

[6] Cole, R.H., Theory of Ordinary Differential Equations, Appleton-CenturyCrofts, [1968].

[7] Rabinowitz, P., Some Global Results for Nonlinear Eigenvalue Problems, Journal of Functional Analysis, 7, 487-513, [1971].

[8] Pazy, A., Semigroups of Linear Operators and Applications to Partial Differential Equations, Appl. Math. Sci., Springer-Verlag, [1983].

[9] Wu, J., Theory and Applications of Partial Functional Differential Equations, Appl. Math. Sci., 119, Springer, [1996]. 
[10] Kaplan, W., Elements of Differential Equations, Addison-Wesley Publishing Company, Inc., [1964].

[11] Hönig, C.S., Análise Funcional e o Problcma de Sturm- Liouville, São Paulo, Editora Edgard Bliicher: editora da Universidade de São Paulo, [1978].

[12] Krasnoselskii, M.A., Topological Methods in the Theory of Nonlinear Integral Equations, Pergamon Press, [1963].

[13] Huang, W., On Asymptotic Stability for Linear Delay Equations, Differential and Integral Equations, 4, 1303-1310,[1991].

[14] Oliveira, J. C. F., Hopf Bifurcation for Functional Differential Equations, Nonlinear Analysis Theory, Methods \& Applications, 4, 217-229, Pergamon Press, [1980].

[15] Nicola, S.H.J., Sistemas Impulsivos com Retardamento: Soluções Periódicas, tese de doutorado, ICMC - USP, [2000].

[16] Nicola, S.H.J., Ladeira, L.A.C. \& Táboas, P.Z., Periodic solutions of an impulsive differential system with delay: an $L^{p}$ approach, Fields Institute Communications, to appear.

[17] Golubitsky, M. \& Schaeffer, D. G., Singularities and Groups in Bifurcation Theory, vol. I, Appl. Math. Sciences, 51, Springer-Verlag, [1985].

[18] Knops, R. J., Nonlinear analysis and mechanics: Heriot-Watt Symposium, vol. I, Research notes in mathematics, 17, Pitman Publishing Limited, [1977].

[19] Stakgold, I., Greens's Functions and Boundary Value Problems, John Wiley \& Sons, New York, [1979]. 